\title{
IPEM Code of Practice for high-energy photon therapy dosimetry based on the NPL absorbed dose calibration service
}

David J Eaton ${ }^{1,8}$, Graham Bass ${ }^{2,8}$, Paul Booker ${ }^{3,8}$, John Byrne ${ }^{4,8}$, Simon Duane ${ }^{2,8}$, John Frame $^{5,8}$, Mark Grattan ${ }^{6,8}$, Russell AS Thomas ${ }^{2,8}$, Natalie Thorp ${ }^{3,8}$ and Andrew Nisbet ${ }^{7,8}$

${ }^{1}$ Radiotherapy Physics, Guy's and St Thomas' Hospitals, London, UK

${ }^{2}$ National Physical Laboratory, Teddington, UK

${ }^{3}$ Rosemere Cancer Centre, Royal Preston Hospital, Preston, UK

$10 \quad{ }^{4}$ Radiotherapy Physics, Northern Centre for Cancer Care, Freeman Hospital, Newcastle upon Tyne, UK

${ }^{5}$ Edinburgh Cancer Centre, Western General Cancer Centre, Edinburgh, UK

${ }^{6}$ Radiotherapy Physics, Northern Ireland Cancer Centre, Belfast Health and Social Care Trust, Belfast, UK

157 Department of Medical Physics \& Biomedical Engineering, University College London, London, UK

${ }^{8}$ Institute of Physics and Engineering in Medicine (IPEM) working party, York, UK

Email: davideaton@nhs.net

Abstract

1. Introduction

2. Scope

$25 \quad 3$. Notes on reference conditions

4. Calibration of the secondary standard at NPL

4.1 General formalism

4.2 Calibration procedure at NPL

5. Calibration of a field chamber in a local beam

5.1 Calibration coefficients for the secondary standard in the local beam

5.2 Correction of chamber response for influence quantities

5.3 Cross-calibration of secondary standard and field chamber in the local beam

6. Reference output measurement in a local beam

6.1 Measurement conditions for determination of reference dose in a phantom

6.2 Calibration of the treatment unit in terms of absorbed dose

7. Determination of dose in non-reference conditions

7.1 Large field non-reference conditions

7.2 Small field non-reference conditions

Acknowledgements

40 Appendix I. List of symbols and abbreviations

Appendix II. Use of TPR 20,10 as a beam quality specifier

Appendix III. Uncertainties 24

Appendix IV. Ion recombination corrections 
Appendix V. Recommendations for chamber positioning and calibration of linac output 27 45 Appendix VI. Recommendations for practical small field measurements Appendix VII. Worked examples for application of the COP References 


\begin{abstract}
The 1990 code of practice (COP), produced by the IPSM (now the Institute of Physics and Engineering in Medicine, IPEM) and the UK National Physical Laboratory (NPL), gave instructions for determining absorbed dose to water for megavoltage photon (MV) radiotherapy beams (Lillicrap et al. Phys. Med. Biol. 199035 1355-60). The simplicity and clarity of the 1990 COP led to widespread uptake and high levels of consistency in external dosimetry audits. An addendum was published in 2014 to include the non-conventional conditions in Tomotherapy units. However, the 1990 COP lacked detailed recommendations for calibration conditions, and the corresponding nomenclature, to account for modern treatment units with different reference fields, including small fields as described in IAEA TRS483 (International Atomic Energy Agency, Vienna 2017).
\end{abstract}

This updated COP recommends the irradiation geometries, the choice of ionisation chambers, appropriate correction factors and the derivation of absorbed dose to water calibration coefficients, for carrying out reference dosimetry measurements on MV external beam radiotherapy machines. It also includes worked examples of application to different conditions.

The strengths of the 1990 COP are retained: recommending the NPL2611 chamber type as secondary standard; the use of tissue phantom ratio (TPR) as the beam quality specifier; and NPL-provided direct calibration coefficients for the user's chamber in a range of beam qualities similar to those in clinical use. In addition, the formalism is now extended to units that cannot achieve the standard reference field size of $10 \mathrm{~cm} \times 10 \mathrm{~cm}$, and recommendations are given for measuring dose in non-reference conditions.

This COP is designed around the service that NPL provides and thus it does not require the range of different options presented in TRS483, such as generic correction factors for beam quality. This approach results in a significantly simpler, more concise and easier to follow protocol.

\title{
1. Introduction
}

Several standard protocols or codes of practice (COPs) are available to determine absorbed dose to water in clinical radiotherapy beams (Almond et al. 1999, DIN 2008, IAEA 2000, 2017, NCS 2012, Castellano et al. 2016 etc.). These all trace a calibration chain from a national or regional standards laboratory through to the clinical unit in the local centre. In 1990, the IPSM (now the Institute of Physics and Engineering in Medicine, IPEM) and the UK National Physical Laboratory (NPL) produced a simple COP based on absorbed dose to water for megavoltage (MV) beams (Lillicrap et al. 1990). Calibration coefficients were provided for a range of beam 
qualities similar to those in clinical use, so that generic correction factors for beam quality were the use of tissue phantom ratio (TPR) as the beam quality specifier.

Similar COPs differ in their choice of reference beam quality at which the SS chamber is calibrated and in how the beam quality of the clinical beam relates to this. In the North American COP TG-51 (Almond et al. 1999), the reference beam quality is ${ }^{60}$ Co radiation, and chamber specific beam quality correction factors for other beams are taken from data tables for different chamber types. TG-51 also uses the percentage depth dose at $10 \mathrm{~cm}$ deep, \%dd(10), as the beam quality specifier. The international COP TRS398 (IAEA 2000) includes the options of direct chamber calibration in a suitable accelerator beam, or from ${ }^{60} \mathrm{Co}$ radiation using either experimental or theoretical (generic tabulated) beam quality correction factors. The isocentric dose ratio at $10 \mathrm{~cm}$ and $20 \mathrm{~cm}$ deep, $\mathrm{TPR}_{20,10}$, is used as the beam quality specifier, and direct calibration is recommended to reduce uncertainties.

The simplicity and clarity of the 1990 COP led to widespread uptake across the UK, and ${ }_{2}$ as a probable direct consequence, external dosimetry audits over the last 20 years have shown very high levels of consistency and accuracy (Eaton et al. 2015a, Eaton et al. 2015b, Thomas et al. 2017). However, the 1990 COP lacked detailed recommendations for equipment calibration conditions and the nomenclature to account for various modern treatment units with different reference fields, including small fields as described in TRS483 (IAEA 2017).

This updated COP retains the strengths of the 1990 COP, recommending a specific chamber type as SS chamber, use of TPR as the beam quality specifier, and NPL-provided direct calibration coefficients for a wide range of beam qualities. Calibration coefficients for the local clinical beam can be derived directly from the calibration certificate provided by the NPL using the measured TPR, as previously. However, the formalism is now extended to units that cannot achieve the conventional reference field size of $10 \mathrm{~cm} \times 10 \mathrm{~cm}$, and recommendations are given both for adjusting machine calibration and for measuring dose in such conditions. For clarity and consistency with the contemporary formalisms, subscripts and superscripts will be used as qualifiers to identify explicitly the meaning of terms except in cases where there is no ambiguity and the use of all qualifiers makes the expression cumbersome. A glossary of terms is provided in Appendix I. Clinics in the UK were previously recommended by IPEM to use the 1990 COP (and addendums), and are now recommended to use this COP. Within the UK
Deleted: Comparisons between different COPs have generally shown variations in dose under reference conditions less than $0.5 \%$ (e.g. Huq et al. 2018), but small variations in

Deleted: ,

Deleted: , which is probably a direct consequence 


\section{Scope}

This IPEM 2020 COP supersedes both the 1990 COP (Lillicrap et al. 1990) and the main body of the 2014 Tomotherapy addendum (Thomas et al. 2014), and formally defines the reference dosimetry for flattening filter free (FFF) beams as outlined in Topical Report 1 (Budgell et al. 2016). Users should refer to those reports only for background to the data contained herein. The COP does not cover MR-linacs where the published scientific work is not yet sufficiently mature, or specific data for Gamma Knife units. However, the formalism may be extended to these units as a later addendum.

For MV photon beams in the quality index (QI) range provided by NPL, currently $\operatorname{TPR}_{20,10}$ (10 $\mathrm{cm} \times 10 \mathrm{~cm}$ ) of 0.568 to 0.800 , this COP provides:

1. Specification of reference conditions in both treatment units capable of $10 \mathrm{~cm} \times 10 \mathrm{~cm}$ field size and treatment units that cannot achieve this;

2. Specification of chambers for secondary standard (SS) use and local measurements;

3. Instructions for determining a SS calibration coefficient;

4. Specification of chamber correction requirements;

5. Instructions and worked examples for determining a field chamber (FC) calibration coefficient;

6. Instructions for determining absorbed dose to water under either standard reference conditions or machine specific reference (msr) conditions. These instructions would normally also be used for adjusting the output of an MV therapy machine;

7. Instructions and worked examples for measuring absorbed dose under non-reference conditions.

Consideration of dose-to-medium calculations by treatment planning systems or otherwise is not covered by this COP. Further discussion may be found elsewhere (e.g. Ma and $\mathrm{Li}$ 2011, Andreo 2015). All chamber calibrations are considered in terms of dose-to-water.

Guidance is not given in the COP regarding who should perform or take responsibility for measurements, or the role of independent checks in definitive calibrations. Users should refer to other IPEM guidance for these areas (e.g. IPEM 2018, appendix A).

\section{Notes on reference conditions}

Three conditions are defined: 
- Absolute dosimetry is based on water or graphite calorimetry and for the purpose of this COP is that measured by the primary standard at NPL under laboratory conditions. It is used to provide a calibration factor for SS detectors under reference conditions.

- Reference dosimetry refers to the measurements of absorbed dose to water under reference conditions using the SS detector or with an FC cross calibrated against the SS under reference conditions.

- Relative dosimetry refers to the measurement of dose in conditions other than the reference conditions which may be done using either of the above-mentioned detectors.

The principle of a reference condition is that it should be a reproducible region of dose that can be repeatedly delivered by a therapy unit and be used as the reference for measurement of dose in other fields. The reference conditions are defined by the combination of the field size, the depth of the measurement, the distance of the detector or surface from the source, the phantom in which the measurement is made, the temperature and pressure of the air in the detector, the amount of phantom surrounding the detector that will influence scatter and attenuation that in turn defines the dose delivered to the point of measurement. Ideally the conditions will be stable dosimetrically with a field size large enough to ensure lateral charged particle equilibrium (LCPE) and ensure that the reference detector used to measure dose within it does not perturb the field to an extent that prevents the measurement from representing the dose in the absence of the detector.

Where the ideal reference conditions cannot be achieved, for example in equipment that cannot deliver a $10 \mathrm{~cm} \times 10 \mathrm{~cm}$ field, then a machine specific reference $(\mathrm{msr})$ condition can be defined (Alfonso et al. 2008). In situations where even the largest beam is difficult to measure and be taken as a reference beam, a planned dose volume can be defined that will serve as a reference field for equipment calibration. Alfonso et al. (2008) introduced this idea as a plan class specific reference (pcsr) field. Often the term beam and field are used interchangeably but in discussing msr and pcsr it is useful to make the distinction that a beam is always a single exposure and when reference dosimetry is discussed it refers to a static single exposure. A field is a region of dose and may be the result of a single static beam exposure or the composite of multiple beams such as in a pcsr field. This COP makes no recommendations on the use of pcsr fields for reference dosimetry other than for calibration of treatment unit monitor chambers, but this may be added in a future addendum. 


\section{Calibration of the secondary standard at NPL}

\subsection{General formalism}

The 1990 COP related the absorbed dose (D) to the chamber reading $(R)$ and the calibration coefficient $\left(\mathrm{N}_{\mathrm{D}}\right)$ through:

$$
D=R N_{D}
$$

This expression, however, contains no information about the calibration conditions such as beam quality, or the superscripts and subscripts introduced by Alfonso et al. (2008) for small or non-conventional fields. Generalising this and using contemporary nomenclature used in TRS398 (IAEA 2000) the absorbed dose to water, $D_{w}$, in a local clinical beam quality, $Q$, using a chamber calibrated by a standards laboratory in a reference beam quality, $Q_{0}$, can be expressed as:

$$
D_{w, Q}=M_{Q} N_{D, w, Q_{0}} k_{Q, Q_{0}}
$$

where:

$D_{w, Q}=$ absorbed dose to water at the chamber reference point, when the chamber (and any sheath) are replaced with water;

$M_{Q} \quad=$ measured chamber reading, usually in charge $(n C)$, corrected for influence quantities such as temperature, pressure and ion recombination. Note that in line with IAEA COPS, M is now used instead of $R$ throughout this COP;

$N_{D, w, Q_{0}}=$ chamber calibration coefficient for the reference beam quality $\left(Q_{0}\right)$, to convert the corrected reading to absorbed dose to water;

$k_{Q, Q_{0}}=$ chamber-specific correction factor to account for any differences in chamber response between the local beam quality $(Q)$, and reference beam quality $\left(Q_{0}\right)$.

Differences in beam quality response of a chamber can arise from either the beam energy (spectrum) or the field size, $f$. The IAEA COP TRS483 (IAEA 2017), in common with the IPEM 2014 Tomotherapy addendum (Thomas et al. 2014), expands the notation for both influences. The local beam is also defined as the machine-specific reference field ( $m s r)$, which is particularly relevant for treatment machines that cannot establish the conventional $10 \mathrm{~cm} \times 10$ $\mathrm{cm}$ reference field $\operatorname{size}^{1}\left(f_{\text {ref }}\right)$ :

\footnotetext{
${ }^{1}$ Note that for beams with non-uniform profiles (e.g. FFF) the determination of field size requires a different method of beam normalisation to that used in flattened beams (e.g. Budgell et al 2016)
} 


$$
D_{w, Q_{m s r}}^{f_{m s r}}=M_{Q_{m s r}}^{f_{m s r}} N_{D, w, Q_{0}}^{f_{r e f}} k_{Q_{m s r}, Q_{0}}^{f_{m s r} f_{r e f}}
$$

where: $D_{w, Q_{m s r}}^{f_{m s r}}$ is the absorbed dose to water in the msr beam quality and field size, and other factors follow the nomenclature described above.

This expression, however, can be simplified when the chamber is calibrated in beam qualities that are spectrally similar $\left(Q_{m s r}\right.$ is similar to $\left.Q_{0}\right)$ and the local field size is the same as the reference (msr equals ref). A superscript is also added to distinguish between a chamber calibrated directly by NPL, i.e. a secondary standard (SS), and a chamber used locally, a field chamber $(F C)$ which has been calibrated by cross-calibration. Therefore, the formalism for a SS chamber does not require a chamber-specific quality correction factor and is given by:

$$
D_{w, Q}=M_{Q} N_{D, w, Q}^{S S}(Q I)
$$

When referring to the calibration coefficient, the beam quality index $(Q I)$ should be given in brackets as shown (see worked examples in Appendix VII). In reduced notation, this expression is equivalent to that given in the 1990 COP (Lillicrap et al. 1990).

For units that cannot achieve the conventional reference (ref) field (e.g. Tomotherapy, CyberKnife, Gamma Knife), or the beam quality is not covered by the NPL calibration curve (e.g. flattening filter free, FFF beams), a machine-specific reference field (msr) should be used and a correction factor, $k^{s s}$, should be included:

$$
D_{w, Q_{m s r}}=M_{Q_{m s r}} N_{D, w, Q_{0}}^{S S}\left(Q I_{0}\right)^{S S}
$$

where: $k^{S S} \equiv k_{Q_{m s r}, Q_{0}}^{f_{m s r}, f_{r e f}}$ as given in equation (3), expresses the correction required for the SS chamber between the NPL calibration and the local field; and $Q_{0}$ refers to the local reference beam quality.

In this COP, $k^{S S}$ chamber-specific quality correction factors are used to account for spectral differences between the msr and the ref field, whereas different field sizes are accounted for by converting the $Q$ I to an equivalent value for the reference field (see section 5.1 ), to determine $N_{D, w, Q}^{S S}$. Appropriate values for $k^{S S}$ are listed in Table 1.

\subsection{Calibration procedure at NPL}

The NPL primary standard is a graphite calorimeter, which is used to calibrate at least three NPL reference standards in a graphite phantom, these reference standards being ionisation 
chambers of type NPL2611 (and all previous versions)2. The absorbed dose to graphite calibrations are converted to absorbed dose to water using factors determined by Burns (1994) and Nutbrown et al. (2002) ${ }^{3}$. The reference standard calibration was originally determined in seven beams from the NPL research linac and in ${ }^{60} \mathrm{Co}$ from which a cubic polynomial fit of the calibration coefficient as a function of beam quality index, $T P R_{20,10}$, was calculated. The fit was used to generate the reference standard calibration coefficients for the beam qualities produced by the NPL Elekta linac. These beams include a conventional flattening filter and this approach was verified by Pearce et al. (2011). At NPL the user chamber is now calibrated in terms of absorbed dose to water by direct comparison with the reference chambers in a water phantom, using a range of beam qualities from the Elekta linac and a ${ }^{60} \mathrm{Co}$ beam. Further discussion on the use of $\mathrm{TPR}_{20,10}$ is given in Appendix II.

Throughout the calibration, the axis of the ionisation chamber is positioned perpendicular to the axis of the beam. The reference point for the chamber is taken to be on the chamber axis, 5 $\mathrm{mm}$ from the tip of a chamber of type NPL2611. The NPL-supplied waterproof sheath has an engraved line at this point. Therefore, any physical offset of the effective measurement point is explicitly accounted for during the calibration.

Calibration conditions are $10 \mathrm{~cm} \times 10 \mathrm{~cm}$ field size, $100 \mathrm{~cm}$ source-to-chamber distance (SCD) and depth in water of $5 \mathrm{~cm}$ (quality index $0.57-0.75,{ }^{60} \mathrm{Co}$ to nominal accelerating potential 10 $\mathrm{MV})$ or $7 \mathrm{~cm}(0.76-0.80$, nominal $>10 \mathrm{MV})$.

The chamber in its waterproof sheath is placed at the required depth in a water phantom. At each beam quality the reference standards and the chamber under test are alternately placed in the water phantom with their geometric centres at the same focal distance on the central axis of the photon beam. Their responses are compared via linac monitor chambers except for the ${ }^{60} \mathrm{Co}$ gamma-ray beam where the use of a monitor chamber is unnecessary.

In most cases the response of the chamber under test is measured using the same measuring

\footnotetext{
${ }^{2}$ The 1990 code specifies the use of NPL-designed NE2561 chamber. Its successor, the NE2611, has been manufactured by NPL since Nuclear Enterprises stopped production and is now referred to as the NPL2611. The two chamber types are radiologically equivalent for MV photon dosimetry: any difference in their energy dependence has been found to be less than $0.1 \%$ and below the level that can be detected in NPL absorbed dose calibrations.

${ }^{3}$ Changes to the UK national primary standards of air kerma and absorbed dose, effective from $1^{\text {st }}$ of September 2019, were implemented following the UK adoption of the recommendations of the International Commission on Radiation Units Report No. 90 (ICRU 2016) and the introduction of new or re-evaluated primary standards. The NPL publication IR 55 (Bass et al. 2019) summarises the changes and the effects of these changes on the calibration coefficients of secondary standards.
} 
circuit as that used for the reference standards. Additional measurements with the chamber connected to the user electrometer, if supplied, are made in the ${ }^{60} \mathrm{Co}$ gamma-ray facility.

The calibration certificate contains calibration coefficients for the beam qualities present on the NPL Elekta linac and ${ }^{60} \mathrm{Co}$, and also a cubic polynomial fit as a function of TPR ${ }_{20,10}$, from which the user can derive values for local beam qualities within this range. No specific frequency of recalibration is recommended, this should be determined locally in conjunction with quality control checks of stability. However, the current practice of three-yearly recalibration is reasonable, unless chambers have demonstrated long-term stability over five years or more (IAEA 2000, Thomas et al 2017).

\section{Calibration of a field chamber in a local beam}

\subsection{Calibration coefficients for the secondary standard in the local beam}

The preferred method of establishing traceability of absorbed dose to water to the primary standard is via a calibrated SS chamber. The NPL2611 or predecessor chambers remain the recommended SS chamber. Other chambers may be used as an FC. Any ionisation chamber meeting the chamber specifications found in McEwen et al. (2014) is suitable but such FCs should only be used if the field-dependent quality correction factor for their type is wellcharacterised. Regular constancy checks should be performed on all chambers.

The chamber must be used with a traceable calibrated electrometer. Care should also be taken to ensure that the polarising potential on the chamber is the same as that during the NPL calibration, typically central electrode positive with respect to graphite cap. It is recommended that the same voltage be used for a specific chamber throughout the calibration chain.

It is essential to understand how the voltage generated by the electrometer is applied to the chamber. Note that the use of an adaptor between different connector types on electrometer and chamber may change the polarity. Owing to the possible combinations of chamber types and connectors, and even variations among electrometers apparently of the same type, users should refer to the manufacturer's operating manual for their particular instrument to check that the polarisation is being set correctly. IPEM guidance on the use of electrometers for dosimetry systems has been published previously by Morgan et al. (2000).

The procedure for determination of $N_{D, w, Q}$ and $k^{S S}$ from $\mathrm{TPR}_{20,10}$ may follow two approaches:

Method A. For equipment where a $10 \mathrm{~cm} \times 10 \mathrm{~cm}$ reference field is achievable: 
- Measure the local quality index $(Q I), \operatorname{TPR}_{20,10}(10 \mathrm{~cm} \times 10 \mathrm{~cm})$, which is the dose ratio in a static $10 \mathrm{~cm} \times 10 \mathrm{~cm}$ field size at the detector, at an SCD of $100 \mathrm{~cm}$, and depths of $10 \mathrm{~cm}$ and $20 \mathrm{~cm}$.

- Determine the absorbed dose to water calibration coefficient for the SS chamber, $N_{D, w, Q}^{S S}(Q I)$ using the curve fit expression provided in the NPL certificate.

- Determine any chamber-specific quality correction factor for the $\mathrm{SS}, \mathrm{k}^{\mathrm{SS}}$ from Table 1 ,

Method B. For treatment machines where a $10 \mathrm{~cm} \times 10 \mathrm{~cm}$ reference field is not achievable (but the reference field is not considered small, see Appendix $\mathrm{VI}$ ):

- Select an msr field as close to $10 \mathrm{~cm} \times 10 \mathrm{~cm}$ as possible and satisfying the conditions for reference dosimetry given in section 3 .

- Measure $\mathrm{TPR}_{20,10}$ ( $\mathrm{msr}$ ): the dose ratio in the msr field size specified by Table 1 , using a typical SCD for that unit, at depths of $10 \mathrm{~cm}$ and $20 \mathrm{~cm}$.

- Convert this value to give the quality index in a hypothetical $10 \mathrm{~cm} \times 10 \mathrm{~cm}$ field, TPR 20,10 $(10 \mathrm{~cm} \times 10 \mathrm{~cm})$, using the equation derived by Palmans (2012):

$$
Q I=T P R_{20,10}(10 \times 10)=\frac{T P R_{20,10}(s)+d_{1(10-s)}}{\left.1+d_{2(10-s}\right)}
$$

where: $s$ is the equivalent square collimated field size at isocentre, and $d_{1}=d_{2}=$ $16.5 \times 10^{-3}$ was the best fit value where the equivalent square of the msr field size is in the range of $4-12 \mathrm{~cm}$.

Equivalent square field sizes can be calculated using $\frac{4 . \text { area }}{\text { perimeter }}$ (for a rectangle of moderate elongation), 0.9 times the equivalent diameter for a circle, or otherwise as described in Appendix A of BIR (1996). Worked examples are given in Appendix VII.

- Determine $N_{D, w, Q}^{S S}(Q I)$ and $k^{S S}$ from the $\mathrm{TPR}_{20,10}$ as for method A above.

TPR measurements should be made in water or water-equivalent material, with the phantom extending at least $5 \mathrm{~cm}$ outside the beam edges and beyond the chamber centre along the beam axis. Readings should be corrected for any differences in chamber temperature and pressure, but for ion recombination losses only if substantial (see Appendix IV). The same exposure (monitor units, MU, or time) should be used for all readings.

Note that the calibration coefficients determined at the NPL apply to measurements made in 
beams produced by linacs with flattening filters (WFF) ${ }^{4}$. These should be corrected for chamber-specific quality dependence when the SS is used for measurements in an FFF beam (or any other situation where the beam qualities cannot be considered spectrally similar). Uncertainties for this calibration are given in Appendix III.

\begin{tabular}{|c|c|c|}
\hline Unit & $\begin{array}{l}m s r \text { static } \\
\text { field size } \\
(\mathrm{cm} \times \mathrm{cm})\end{array}$ & $\begin{array}{c}k^{S S} \\
\text { (chamber type, } \\
\text { reference) }\end{array}$ \\
\hline $\begin{array}{l}\text { TomoTherapy (\& } \\
\text { Radixact) }\end{array}$ & $5 \times 10$ (static) & $\begin{array}{l}1.000 \pm 0.03 \\
\text { (NPL2611, } \\
\text { Thomas et al. } \\
2014)\end{array}$ \\
\hline $\begin{array}{c}\text { CyberKnife } \\
\text { (circular collimators } \\
\text { e.g. VSI) } \\
\text { (micro-MLC e.g. M6) }\end{array}$ & $\begin{array}{l}6 \text { circle } \\
10 \times 10\end{array}$ & $\begin{array}{c}1.000 \\
(\mathrm{NPL} 2611, \text { IAEA } \\
\left.2017^{5}\right)\end{array}$ \\
\hline $\begin{array}{c}\text { Flattening filter free } \\
\text { (FFF) beams (QI < } \\
0.75 \text {, e.g. Varian \& } \\
\text { Elekta) }\end{array}$ & $10 \times 10$ & $\begin{array}{c}0.997 \pm 0.03 \\
\text { (NPL2611, Budgell } \\
\text { et al. 2016) }\end{array}$ \\
\hline $\begin{array}{l}\text { Add-on SRS cones } \\
\text { or micro-MLC (e.g. } \\
\text { Elekta Apex) }\end{array}$ & $\begin{array}{l}\text { As close to } 10 \\
\times 10 \text { as } \\
\text { possible }\end{array}$ & $\mathrm{n} / \mathrm{a}$ \\
\hline
\end{tabular}

Table 1 - Chamber-specific quality correction factors for specialist treatment machines. N.B. Gamma Knife units will be covered in a separate addendum.

\subsection{Correction of chamber response for influence quantities}

Raw readings $\left(\mathrm{M}_{\mathrm{raw}}\right)$ taken during measurements with a chamber-electrometer combination need to be corrected for the following influence quantities to give $M_{Q}$ as listed in the formalism

${ }^{4}$ The term WFF is used in this COP to refer to non-FFF beams. In the Tomotherapy addendum (Thomas et al. 2014) this was referred to as conventional flattening filter beams. Both terms refer to the same situation and WFF is used here for consistency with TRS483 (IAEA 2017).

${ }^{5}$ Comparing tables 12 and 13 in TRS-483 suggests a value of 1.000 , which includes a generic volume averaging correction factor from table 32 of 1.002 for this chamber and treatment unit. If this factor is included with the corrections for other influence quantities, then a value of 0.998 should be used for $k^{\text {ss }}$. For other FFF beams, the correction ( $\mathrm{k}_{\mathrm{vol}}$ ) can be taken as unity as described in section 5.2 below. 
equations. Some of these values will be negligible, or incorporated into the cross-calibration for the FC, but are included here for completeness.

$$
M_{Q}=M_{\text {raw }} k_{\text {elec }} k_{T P} k_{h} k_{\text {ion }} k_{\text {pol }} k_{\text {vol }}
$$

where:

$M_{\text {raw }}$ are readings taken once electronics have warmed-up and stabilised, and the chamber has reached thermal and charge equilibrium. Small amounts of leakage may be accounted for by automatic background correction, but excessive leakage $(>1 \%)$ should be investigated as an indication of potential malfunction;

$k_{\text {elec }}$ is the charge calibration coefficient for the electrometer, since this is calibrated by NPL separately from the SS chamber. Depending on the type of electrometer, $\mathrm{k}_{\text {elec }}$ may be a single correction factor reported in the calibration certificate or calculated by the user as the product of the reported range and non-linearity correction factors. This COP recommends that the FC and electrometer are ideally considered as a single instrument, so $\mathrm{k}_{\text {elec }}$ is not required for the $\mathrm{FC}^{6}$;

$\mathrm{k}_{\mathrm{TP}}$ is the temperature-pressure correction factor, accounting for any difference in mass of air within the chamber active volume, according to the ideal gas law, compared to reference temperature $\left(T_{0}\right)$ of $20^{\circ} \mathrm{C}$ and reference pressure $\left(P_{0}\right)$ of 1 atmosphere $(1013 \mathrm{mbar}$ or $760 \mathrm{~mm}$ $\mathrm{Hg})$. Temperature $\left({ }^{\circ} \mathrm{C}\right)$ should be measured for the phantom not the surrounding room air;

$$
k_{T P}=\frac{(273.2+T) P_{0}}{\left(273.2+T_{0}\right)^{P}}
$$

$\mathrm{k}_{\mathrm{h}}$ is the humidity correction factor, compared to the reference of $50 \%$, which can be taken as unity in the range $20-70 \%$ relative humidity. Outside of this range, NPL can provide advice;

$\mathrm{k}_{\mathrm{ion}}$ is the correction factor for losses because of ion recombination at finite collection voltages ${ }^{7}$. Correction is essential in pulsed radiation beams and may be substantial in high-dose rate beams such as FFF. Correction factors should be verified experimentally, for example by using the two-voltage technique with a voltage ratio of 3 or greater, or using a verified empirical

\footnotetext{
${ }^{6}$ Where this is not practical, for example equipment distributed across multiple locations, crosscalibrations should be performed to determine FC electrometer factors. The same chamber and measurement setup should be used with the chamber connected to each electrometer in turn, ensuring stabilisation and minimal leakage. Substitution should be repeated at least twice. Factors should be consistent between different energies, and similar to other electrometers of the same type.

${ }^{7}$ The ion recombination factor has various symbols in different COPs, including $k_{s}$ in TRS398/483 and $P$ ion, and these are all equivalent, but kion is used here for clarity.
} 
$\mathrm{k}_{\mathrm{pol}}$ is the correction factor for polarity effects, which is negligible for the NPL2611 chamber and only significant for small volume chambers. NPL do not apply any correction for polarity, so as long as the polarity is kept constant during the calibration process, then any effects are incorporated into the cross-calibration.

$\mathrm{k}_{\mathrm{vol}}$ is the beam non-uniformity correction factor, which accounts for volume averaging and perturbation of secondary electron fluence, in a non-uniform beam profile such as FFF. For a thimble chamber, the volume averaging effect is approximately proportional to the square of the length of the sensitive volume. For the NPL2611 chamber in 6 MV FFF or 10 MV FFF beams, this correction factor can be taken to be unity, with an uncertainty of $0.2 \%$ (Budgell et al. 2016). Correction factors for Farmer-type (2571) chambers may be of the order of $1 \%$. Any effects for FC may be incorporated into the calibration coefficient if conditions are the same between cross-calibration and reference output measurement, but should be considered separately for non-reference conditions.

\subsection{Cross-calibration of secondary standard and field chamber in the local beam}

The preferred method to determine a calibration coefficient for the FC is through a crosscalibration with the SS chamber (also called intercomparison). When the two chambers are exposed in the same local (msr) beam, the dose to both chambers can be equated to give the FC calibration coefficient:

$N_{D, w, Q_{m s r}}^{F C}\left(Q I_{m s r}\right)=N_{D, w, Q_{0}}^{S S}\left(Q I_{0}\right) \frac{M_{Q_{m s r}}^{S S}}{M_{Q m s r}^{F C}} k^{S S}$

where $k^{s S}$ corrects for the change in response of the SS chamber between the reference beam quality $\left(\mathrm{Q}_{0}\right)$ and the msr beam quality. For flattened beams (WFF), or otherwise where $k^{s s}=1$, this equation can be simplified as follows:

$$
N_{D, w, Q}^{F C}(Q I)=N_{D, w, Q}^{S S}(Q I) \frac{M_{Q}^{S S}}{M_{Q}^{F C}}
$$

Cross-calibration should be performed either by sequential substitution or by simultaneous irradiation side-by-side, in either a water phantom or a near-water-equivalent solid phantom. If liquid water is used, the NPL2611 chamber should be waterproofed using the thin plastic sheath used during the calibration by NPL. Alternatively, Perspex block phantoms have been used for many years with acceptable accuracy for relative measurements (Lillicrap et al. 1990). Figure 1 shows an example of these blocks. The phantom dimensions should extend at least $5 \mathrm{~cm}$ 
beyond each field edge and at least $5 \mathrm{~cm}$ beyond the chamber centre along the beam axis to provide sufficient scatter conditions.

The reference points of the chambers should be positioned $5 \mathrm{~cm}$ deep in a static field at a typical SCD. Ideally the depth of cross-calibration should be the same as the depth of absolute dosimetry (by NPL, section 4.2) and reference dosimetry (for calibration, section 6.2). However, using a depth of $5 \mathrm{~cm}$ for cross-calibration of all energies is a reasonable and pragmatic approximation. Note that the reference point of thimble ionisation chambers is defined as the point in the chamber where the measured dose is applicable. This is taken by NPL as the chamber centre and no adjustment should be made for the effective point of measurement (EPOM) in the direction of the beam axis. If the EPOM is different in each chamber this difference will be built into the calibration coefficient as the cross-calibration will produce a coefficient that will allow the dose in a SS to be determined at the reference point from a measurement in FC.

The field size (at the chamber position) should ideally be at least $10 \mathrm{~cm} \times(\mathrm{sep}+10) \mathrm{cm}$, where sep is the separation between the chambers for side-by-side irradiation. However, a field size of $10 \mathrm{~cm} \times 10 \mathrm{~cm}$ may be used in WFF or FFF beams for NPL2611 and Farmer-type chambers where sep is $3 \mathrm{~cm}$ or less; and msr field sizes and chambers listed in Table 1, may also be used with minimal differences. If both chambers are of the same model or design, there is no minimum separation, since the influence of one chamber on the other will cancel out. Where they are of different types, sep should exceed the lateral charge particle equilibrium range $\left(r_{L C P E}\right)$ in that beam quality (see Appendix $\mathrm{VI}$ ).
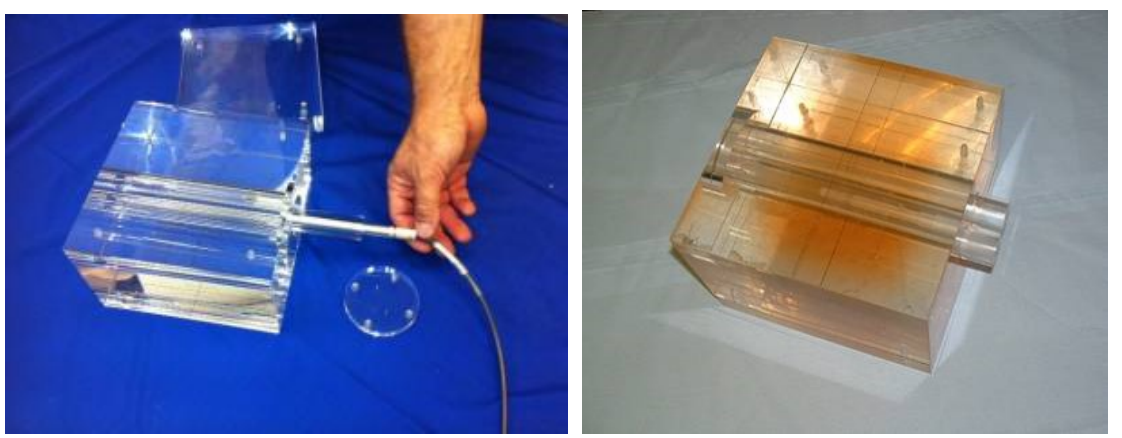

Figure 1 - Perspex cross-calibration phantom commonly used in many centres. Left image courtesy of Phoenix Dosimetry Ltd, Sandhurst, UK.

All equipment should have reached thermal equilibrium before starting measurements. Raw 
chamber readings should be corrected for influence quantities as described in section 5.2 above. Many of these will be constant or included within the cross-calibration for the FC, but factors of unity should be stated for completeness.

For side-by-side irradiation, at least two measurements (of a pair of readings) should be acquired in the first configuration (series $\mathrm{A}$ ), then the positions interchanged, and at least two measurements taken (series $\mathrm{B}$ ). The cross-calibration ratios are given by:

$$
\begin{aligned}
& A=\frac{M_{Q, A}^{S S}}{M_{Q, A}^{F C}} \\
& B=\frac{M_{Q, B}^{S S}}{M_{Q, B}^{F C}}
\end{aligned}
$$

The process of interchange should be followed at least twice obtaining ratios for the two positions (e.g. ABA). The ratio of the chamber readings should be calculated as the geometric mean to reduce the influence of spatial variation during acquisition (Abdel-Rahman et al. 2009):

$$
\frac{M_{Q}^{S S}}{M_{Q}^{F C}}=\sqrt{\overline{A B}}
$$

where: $A$ is the mean of the ratios for series $A, B$ is the mean of the ratios for series $B$. The standard deviation of readings should usually be within $0.3 \%$.

For sequential substitution, at least three readings should be taken for each chamber and the whole process repeated at least once. The ratio of the chamber readings may use the average of each series. The standard Perspex phantom may be used if one hole is filled with a solid Perspex rod, and the other hole aligned to the field centre. It is not normally necessary to use an external monitor chamber in modern linac beams.

For high dose-rate pulsed beams or those with non-uniform profiles (e.g. FFF) calibration by substitution is recommended (following the guidance in Budgell et al 2016), so that all measurements are made with the chamber reference point on the beam central axis. Side-by-

side calibration may only be used if local measurements demonstrate acceptable accuracy. There will be jncreased uncertainty from the effects of positioning a chamber within a dose gradient when off-axis in an FFF beam.

When the SS and FC are cross-calibrated in steep dose gradients the effect of volume averaging should be assessed, though for the NPL2611 chamber in both 6MV FFF and 10MV FFF beams this is of the order of $0.1 \%$ and may be considered negligible (Budgell et al 2016). However, this cannot be assumed for the FC. The ion recombination factors for the two 
chambers may also vary between the two different setup methods and so the cross-calibration derived calibration coefficient for the FC may only be valid at the cross-calibration measurement point, and suitable corrections applied where differences are significant.

Treatment machine electronic servos may be slow in controlling high dose rate beam delivery and so for both methods of cross-calibration the irradiation times should be long enough to ensure beam stability and accuracy of beam output.

\section{Reference output measurement in a local beam}

Measurement of absorbed dose using the FC in the local reference field is given by:

$$
D_{w, Q}=M_{Q} N_{D, w, Q}^{F C}(Q I)
$$

Note that chamber readings should be fully corrected for influence quantities such as temperature, pressure and ion recombination, as described in section 5.2, although some of these will be negligible.

\subsection{Measurement conditions for determination of reference dose in a phantom}

A liquid water medium around the chamber is preferred for the highest accuracy applications (IAEA 2000). However, use of a solid phantom material will usually be more practical for routine measurements (IAEA 2017). There is insufficient data to recommend a certain purity of water, so no guidance is given in this regard. Blocks of solid near-water-equivalent material are quick and easy to setup with high positional reproducibility, and do not require waterproof chambers. Note that both solid and liquid phantoms may take several hours to reach thermal equilibrium with their surroundings, therefore the temperature should be checked to confirm equilibrium before starting any measurements. Materials such as wood have previously been used, but are susceptible to variability between samples, and porous materials may change shape and composition, so these are not recommended.

For absorbed dose measurement, the water-equivalence of any solid phantom should be established in advance (Lillicrap et al. 1990, Thomas et al. 2014, Budgell et al. 2016), and at least at the time of definitive calibrations (IPEM 2018). Where differences in readings taken in the phantom compared to liquid water exceed measurement uncertainties, typically $0.3 \%$, an additional correction factor, $k_{Q}^{w, p h a n t o m}$ should be applied. For epoxy-resin based plastics this

factor will usually be less than $1 \%$, but the user should establish factors for local materials as these can vary even between materials of the same type. Larger corrections are not recommended for reference output measurement, for example when using generic plastics like Perspex. In some cases, it may be necessary to scale the depth in phantom to the equivalent depth in water based on the ratio of electron densities, but for many near-water-equivalent 
materials these effects will be negligible (IAEA 2017). Liquid water filled mini-phantoms should be checked for window distortions, but these will usually also be negligible (Kinoshita et al. 2019).

The phantom material should extend at least $5 \mathrm{~cm}$ outside the field edges and $5 \mathrm{~cm}$ beyond the chamber along the beam axis. The depth of measurement between the phantom surface and the chamber position depends on the quality index: for $0.57-0.75\left({ }^{60} \mathrm{Co}\right.$ to nominal $10 \mathrm{MV}$ ), the depth should be $5-10 \mathrm{~cm}$; and for $0.76-0.80$ (nominal >10 MV) the depth should be 7-10 $\mathrm{cm}$. See Appendix $\mathrm{V}$ for further discussion and rationale for these recommendations.

\subsection{Calibration of the treatment unit in terms of absorbed dose}

Appropriate positioning of the ion chamber for reference dosimetry involves two different considerations: (i) chamber position for accurate and practical measurement of reference dose, and (ii) the reference position for calibration of the unit (e.g. the linac output is adjusted to give a dose of 1 cGy per MU at this point, within appropriate tolerances, see e.g. IPEM (2018). To facilitate consistency between centres, this COP makes recommendations for machine calibration conditions, as well as for measurement conditions, which was not part of guidance in the previous 1990 COP. There is no immediate requirement to change calibration conditions to use this COP, but all centres should seek to adopt the recommendations at a suitable time.

Two options are recommended for machine calibration setup:

1) Calibrate all energies to give $1 \mathrm{cGy}$ per $\mathrm{MU}$, at either $5 \mathrm{~cm}$ deep (up to $10 \mathrm{MV}$ ) or $7 \mathrm{~cm}$ deep (>10 MV), with isocentric positioning (fixed SCD, usually $100 \mathrm{~cm}$ ). This option is consistent with the NPL and cross-calibration setup, along with previous UK COP and addenda;

2) Perform all measurements at $10 \mathrm{~cm}$ deep, with isocentric positioning, and calibrate to either 1 cGy per MU, or to a different value for each energy which is less than $1 \mathrm{cGy}$ per MU (for example to give approximately $1 \mathrm{cGy}$ per $\mathrm{MU}$ at the depth of dose maximum $\left.\left(\mathrm{d}_{\max }\right)\right)$. This depth is recommended in the IAEA COPs. III for a list of uncertainties in the FC measurements.

Ideally the setup for local machine (monitor chamber) calibration would be the same as that used to calibrate the SS chamber at NPL and for cross-calibration, in order to minimise uncertainties in $\mathrm{N}_{\mathrm{D}, \mathrm{w}}$. However, chamber calibration coefficients may be used at measurement depths of 5-10 cm with negligible differences (Almond et al. 1999). For units unable to achieve these conditions, such as a field size of $10 \mathrm{~cm} \times 10 \mathrm{~cm}$, using the msr field size given in Table 1 , will minimise uncertainties in a similar way. 
In general, the minimum field size should extend further than the size of the lateral charged particle equilibrium range $\left(r_{L C P E}\right)$ beyond the outer boundaries of the reference ionization chamber. That is, for a detector of external width $d$, the maximum size of the field should be at least as wide as $2 r_{L C P E}+d$ where $\mathrm{d}$ is the largest dimension presented to the beam (in the plane perpendicular to the beam axis).

\section{Determination of dose in non-reference conditions}

A calibrated FC may be used to make measurements in some fields that do not match the reference conditions. For this to be valid, field-dependent correction factors (in TRS483 this is called a field output correction factor) may need to be determined and applied. Examples of non-reference condition measurements are: individual measurements to investigate patient doses, and relative dose ratios such as PDDs, TPRs, output factors, isodoses etc. For these parameters, where ratios of dose are required by definition, it is possible to use uncalibrated detectors only when it can be shown that the ratio of uncalibrated readings equates to the ratio of dose.

For measurements of dose in non-reference conditions to be valid the chamber response must be corrected if the conditions of the field mean that the response of the chamber changes between the reference and non-reference field. This can happen if the spectrum of the field changes significantly or the field size becomes small enough that the FC perturbation becomes significant.

When calculating dose from measurements all detector corrections should be applied to produce the corrected reading $M_{Q}$ unless it can be shown that the overall effect is negligible. The decision to neglect a correction should not be done repeatedly for a number of corrections without assessing the combined effect. If dose is to be attributed to a specific point the effective point of measurement of the ionisation chamber must be taken into account. For measurements in non-uniform fields, such as wedged or FFF, the chamber should be oriented to minimise the gradient across the chamber.

\subsection{Large field non-reference conditions}

In fields larger than the small field threshold (see Appendix VI for definition), stopping-power ratios and perturbation effects can be assumed to a reasonable accuracy to be independent of depth and field size (Andreo and Brahme 1986). The general form for measuring dose in nonreference fields is then Equation 14 with the application of a chamber-specific quality correction factor, $k_{Q_{\text {clin },}, Q_{\text {ref }}}^{f_{\text {clin }},}$ 
$D_{w, Q_{c l i n}}=M_{Q_{r e f}} N_{D, w, Q_{r e f}}^{F C}\left(Q I_{r e f}\right)^{k} \sum_{Q_{c l i n}, Q_{r e f}}^{f_{\text {clin }}, f_{\text {ref }}}$

where $k_{Q_{c l i n}, Q_{\text {ref }}}^{f_{c l i n}, f_{\text {ref }}}$ accounts for differences in the response of the FC in a clinical (clin) field that is different from the reference field. Where an $\mathrm{msr}$ field is used ref can be replaced by $m s r$. Where there is a plan-class-specific reference field ( $p c s r)$, clin can be replaced by pcsr. For example, Tomotherapy units are often calibrated using a rotational modulated beam with an approximately uniform dose region around chamber. The Tomotherapy addendum (Thomas et al. 2014) recommended a $k_{Q_{p c s r}, Q_{m s r}}^{f_{p c s r}, f_{m s r}}$ value of 1.000 for the A1SL chamber in this pcsr field, referred to as $k^{F C}$ therein.

Values of $k_{Q_{c l i n}, Q_{r e f}}^{f_{\text {clin }}, f_{\text {ref }}}$ for a range of chambers can be found in TRS483 tables 23-27 and it is recommended that these are used. For fields greater than the largest values tabulated $k_{Q_{\text {clin }}, Q_{\text {ref }}}^{f_{\text {clin }}, f_{\text {ref }}}$ should be taken as unity. Values for the Exradin A1SL are not included in this table but it is reasonable to assume unity for fields $\geq 5 \mathrm{~cm}$ in width. Note that the depth of measurement for the factors given in the TRS483 tables is $10 \mathrm{~cm}$ and for any device used at different depths, depth doses should be compared between the detector and a Farmer-type ionisation chamber. Detectors that do not match the Farmer-type ionisation chamber performance should not be used to measure dose.

\subsection{Small field non-reference conditions}

In fields where the cross-calibrated FC is too large to be used in the small field because of excessive perturbation the dose should be calculated from the combination of the reference (or $m s r$ ) field dose and a field output factor (FOF, $\Omega$ ). In the following description it is assumed that a detector has been cross-calibrated in the reference field but the same approach applies when the detector has been cross-calibrated in an $\mathrm{msr}$ field. Dose can be determined from a corrected measurement, $M_{Q}$, by:

$D_{w, Q_{c l i n}}=M_{Q_{r e f}}^{f_{r e f}} N_{D, w, Q_{r e f}}^{F C}\left(Q I_{\text {ref }}\right)^{\Omega_{Q_{c l i n}, Q_{r e f}}^{f_{\text {clin }}, f_{\text {ref }}}}$

where $\Omega_{Q_{\text {clin },}, Q_{\text {ref }}}^{f_{\text {clin }}, f_{\text {ref }}}$ is the FOF relating the clinical field to the reference field.

The FOF must be measured using a detector that is suitable for the small clinical field. If this small field detector is not suitable also for the reference field, for example because it has a strong energy dependence, then a two-step approach using an intermediate field (daisy chaining) should be used. The approach is described further in Appendix VI. It is important to 
remember that an FOF is the ratio of absorbed dose to water in the clinical field to that in the reference field. In conventional broad beams it is reasonable to assume that the ionisation chamber (IC) response can be taken as independent of field size and the FOF can be taken as the ratio of chamber readings. However, in small field dosimetry this independence cannot be assumed to exist and a ratio of readings cannot be taken to represent a ratio of dose. In this situation an FOF requires a chamber-specific output correction factor to be applied to the measured detector reading ratio, and so:

$\Omega_{Q_{\text {clin }} Q_{\text {ref }}}^{f_{\text {clin }} f_{\text {ref }}}=\frac{M_{Q_{\text {clin }}}^{f_{\text {clin }}}}{M_{Q_{\text {ref }}}^{f_{\text {ref }}}} k_{Q_{\text {clin }}, Q_{\text {ref }}}^{f_{\text {clin }}, f_{\text {ref }}}$

or for the case where an intermediate (int) field is used with a different, small, detector (det):

$\Omega_{Q_{\text {clin }}, Q_{\text {ref }}}^{f_{\text {clin }}, f_{\text {ref }}}=\left[\frac{M_{Q_{\text {clin }}}^{f_{\text {clin }}}}{{ }_{Q_{\text {int }}}^{f_{\text {int }}}} k_{Q_{\text {clin }}, Q_{\text {int }}}^{f_{\text {clin }}, f_{\text {int }}}\right] \operatorname{det}\left[\frac{M_{Q_{\text {int }}}^{f_{\text {int }}}}{M_{Q_{\text {ref }}}^{f_{\text {ref }}}} k_{Q_{\text {int }}, Q_{\text {ref }}}^{f_{\text {int }}, f_{\text {ref }}}\right]_{\text {IC }}$

Where an $m s r$ field has been used ref can be replaced by $m s r$ and the description will then match that shown in Appendix VI.

\section{Acknowledgements}

The working party are grateful to the membership of the IPEM radiotherapy special interest group (RTSIG) and the journal reviewers for their helpful comments. 
Appendix I. List of symbols and abbreviations

\begin{tabular}{|c|c|}
\hline Symbol/term & Description \\
\hline COP & Code of practice \\
\hline$D_{w, Q}$ & Absorbed dose to water $(w)$ in a certain beam quality $(Q)$ \\
\hline$f$ & $\begin{array}{l}\text { Radiation field size, usually with a subscript denoting the } \\
\text { field e.g. ref, msr, pcsr etc. }\end{array}$ \\
\hline$F C$ & $\begin{array}{l}\text { Field chamber, usually a Farmer-type or small volume } \\
\text { ionisation chamber }\end{array}$ \\
\hline$F F F$ & $\begin{array}{l}\text { Radiation field which is flattening filter free, i.e. lacking a } \\
\text { conventional flattening filter }\end{array}$ \\
\hline$k_{i}$ & $\begin{array}{l}\text { Generic chamber-specific correction factor for influence } \\
\text { quality (i) e.g. } k_{\text {ion }} \text { or } k_{T P}\end{array}$ \\
\hline $\begin{array}{l}k_{\text {quality } 2, q u a l i t y 1}^{(\text {chamber } 1} \text {,field } 2, \text { field } 1 \\
\end{array}$ & $\begin{array}{l}\text { Generic chamber-specific beam quality and field size } \\
\text { dependent correction factor; includes chamber only when } \\
\text { there would be ambiguity }\end{array}$ \\
\hline $\begin{array}{l}k_{Q_{c l i n}, Q_{\text {ref }}}^{f_{\text {clin }}, f_{\text {ref }}} \\
\end{array}$ & $\begin{array}{l}\text { Field chamber (FC) beam quality correction factor, which } \\
\text { could also relate to } m s r \text { or } p c s r \text { fields }\end{array}$ \\
\hline$k^{S S}$ & $\begin{array}{l}\text { Secondary standard (SS) beam quality correction factor, a } \\
\text { simplification of } k_{Q_{m s r}, Q_{0}}^{f_{m s}, f_{r f}}\end{array}$ \\
\hline isocentric & $\begin{array}{l}\text { Geometry with the chamber positioned at fixed SCD from the } \\
\text { radiation source, usually coincident with the machine } \\
\text { rotation isocentre }\end{array}$ \\
\hline$M_{Q}^{(\text {chamber })}$ & $\begin{array}{l}\text { Measured chamber reading in a certain beam quality (Q), } \\
\text { corrected for influence quantities }\end{array}$ \\
\hline$m s r$ & $\begin{array}{l}\text { Machine specific reference field, used where units cannot } \\
\text { achieve the reference field size }\end{array}$ \\
\hline$N_{D, w, q u a l i t y}^{\text {chamber }}\left(\right.$ field $^{\text {cha }}(Q I)$ & $\begin{array}{l}\text { Generic absorbed dose to water chamber calibration } \\
\text { coefficient, for a certain beam quality (and field size), in } \\
\text { terms of a certain quality index }\end{array}$ \\
\hline$\Omega_{Q_{\text {clin }} Q_{\text {ref }}}^{f_{\text {clin }}, f_{\text {ref }}}$ & $\begin{array}{l}\text { The full scatter field output factor (FOF), which is the ratio } \\
\text { between the absorbed doses in the clinical and reference (or } \\
\text { msr) fields, including the detector specific correction factor, } \\
k_{Q_{\text {clin, }}, Q_{\text {ref }}}^{f_{\text {clin }}, f_{\text {ref }}}\end{array}$ \\
\hline
\end{tabular}




\begin{tabular}{|c|c|}
\hline Symbol/term & Description \\
\hline$P$ & $\begin{array}{l}\text { Pressure, measured using a traceable calibrated barometer } \\
\text { within the local centre, ideally within the treatment room or } \\
\text { at the same elevation }\end{array}$ \\
\hline pcsr & Plan class specific reference field \\
\hline$Q$ & $\begin{array}{l}\text { Beam quality, the unique spectral attribution of a particular } \\
\text { radiation beam, sometimes with a subscript denoting the } \\
\text { field type e.g. reference }(0), \mathrm{msr} \text {, pcsr, etc. }\end{array}$ \\
\hline$Q I$ & $\begin{array}{l}\text { Beam quality index, the numerical value used to determine } \\
\text { the beam quality, in this COP defined by } \\
T P R_{20,10}(10 \times 10) \text { as measured on the central axis. }\end{array}$ \\
\hline$r_{L C P E}$ & Distance of lateral charge particle equilibrium \\
\hline ref & $\begin{array}{l}\text { Reference field, in this COP defined as } 10 \mathrm{~cm} \times 10 \mathrm{~cm} \\
\text { square }\end{array}$ \\
\hline $\begin{array}{l}\text { reference point of } \\
\text { chamber }\end{array}$ & $\begin{array}{l}\text { The reference point of thimble ionisation chambers is } \\
\text { defined as the point in the chamber where the measured } \\
\text { dose is applicable. This may be taken as the chamber centre } \\
\text { or the effective point of measurement (EPOM), depending } \\
\text { on the application as described in the main text. Care should } \\
\text { be taken to ensure consistency during comparison e.g. when } \\
\text { cross-comparing chambers or in calibrating output. }\end{array}$ \\
\hline s, field size & $\begin{array}{l}\text { Radiation field size, the numerical value of } \\
f \text { defined as the calibrated collimator size at level of the } \\
\text { isocentre }\end{array}$ \\
\hline$S C D$ & Source-to-chamber distance \\
\hline$S S D$ & Source-to-surface distance \\
\hline SS & $\begin{array}{l}\text { Secondary standard chamber, in this COP defined as the } \\
\text { NPL2611 }\end{array}$ \\
\hline$T$ & $\begin{array}{l}\text { Temperature }\left({ }^{\circ} \mathrm{C}\right) \text {, measured with a traceably calibrated } \\
\text { thermometer in the phantom material, as a surrogate for the } \\
\text { chamber air temperature }\end{array}$ \\
\hline$T P R_{20,10}(s)$ & $\begin{array}{l}\text { Tissue phantom ratio, of dose measurements (or corrected } \\
\text { chamber readings) in a water-equivalent phantom, at depths } \\
\text { of } 20 \mathrm{~cm} \text { and } 10 \mathrm{~cm} \text {, with constant SCD, and collimated field } \\
\text { size (s) }\end{array}$ \\
\hline
\end{tabular}




\begin{tabular}{l|l}
\hline Symbol/term & Description \\
\hline$W F F$ & $\begin{array}{l}\text { Radiation field with a flattening filter, large enough to flatten } \\
\text { a } 40 \mathrm{~cm} \times 40 \mathrm{~cm} \text { field and significantly change the beam } \\
\text { spectrum }\end{array}$ \\
\hline
\end{tabular}

\section{Appendix II. Use of TPR 20,10 as a beam quality specifier}

Beam quality is the unique spectral attribute of a particular radiation beam that gives rise to a certain chamber response. For the purpose of ion chamber calibration, beam quality is defined to be the properties of the beam at the point of measurement, such as the effective water-toair stopping power ratio that uniquely determine the chamber's absorbed dose-to-water calibration coefficient. It is convenient to represent the beam quality attribute by a single easily measurable numeric value, the beam quality index (QI). The beam QI is therefore the parameter that characterizes beam quality; if two beams have the same beam QI, then the calibration coefficient for a given detector should ideally take the same value in the two beams. Unfortunately, any QI will always be an approximation (c.f. use of half value layer for kilovoltage beams), so further chamber-specific beam quality correction factors $\left(k_{Q}\right)$ may be required. For example, differences between WFF and FFF beams, or between reference fields of different sizes.

IAEA COPs (IAEA 2000 and IAEA 2017), the 1990 COP and this 2020 COP all use TPR 20,10 as the QI. Measurement of TPR is practical and robust, insensitive to measurement errors, independent of electron contamination, and aligns with the majority of modern treatments where beam axes are directed through a single point (the isocentre).

\section{Appendix III. Uncertainties}

Relative standard uncertainties are listed in Table 2 , below for the parameters described in this $\mathrm{COP}$, with expanded values for total uncertainty using a coverage factor $\mathrm{k}=2$, corresponding to 95\% confidence. TRS398 (IAEA 2000) gives the total uncertainty for MV photon calibration as $1.5 \%$, reduced to $1.2 \%$ when the user chamber is calibrated at the primary standard laboratory, and increased to $1.4 \%$ for a FC cross-calibrated in a local beam. The lower values in this COP reflect the reduced uncertainty provided by NPL calibration coefficients, which are applicable across a range of clinical beam qualities. Budgell et al. (2016) also reported an uncertainty of $0.9 \%$ for a FC calibrated following that addendum to the 1990 COP.

\begin{tabular}{|l|l|l|}
\hline Parameter & $\begin{array}{l}\text { Relative standard } \\
\text { uncertainty (\%) }\end{array}$ & Notes \\
\hline
\end{tabular}




\begin{tabular}{|c|c|c|}
\hline$M_{Q}^{S S}$ & 0.3 & $\begin{array}{l}\text { Long term stability of SS chamber } 0.1 \% \text {; short } \\
\text { term repeatability }<0.1 \% \text {; combined influence } \\
\text { factors }\left(k_{i}\right) 0.3 \%\end{array}$ \\
\hline$N_{D, w, Q}^{S S}$ & 0.7 & $\begin{array}{l}\text { Calibration of SS at NPL, as interpolated into } \\
\text { calibration certificate for user beam quality (Q) } \\
\text { N.B. Measurement of TPR } 20,10 \text { uncertainty } 0.4 \% \text {, } \\
\text { but impact on } N_{D, w, Q}^{S S} \text { value }<0.1 \%\end{array}$ \\
\hline$k^{S S}$ & 0.2 & $\begin{array}{l}\text { Beam quality correction factor for SS in msr field } \\
\text { e.g. FFF or Tomotherapy }\end{array}$ \\
\hline$D_{w, Q}^{S S}$ & $\begin{array}{l}0.8 \\
(1.6 \% k=2)\end{array}$ & $\begin{array}{l}\text { Absorbed dose measured in user beam with SS } \\
\text { chamber }\end{array}$ \\
\hline$\frac{M_{Q}^{S S}}{M_{Q}^{F C}}$ & 0.4 & Cross-calibration ratio of SS and FC chambers \\
\hline$M_{Q}^{F C}$ & 0.3 & $\begin{array}{l}\text { Long term stability of } \mathrm{FC} \text { chamber } 0.3 \% \text {; short } \\
\text { term repeatability }<0.1 \% \\
\text { N.B. influence factors for the FC cancel out in } \\
\text { final result, so have no extrinsic uncertainty }\end{array}$ \\
\hline$D_{w, Q}^{F C}$ & $\begin{array}{l}0.9 \\
(1.8 \% \mathrm{k}=2)\end{array}$ & $\begin{array}{l}\text { Absorbed dose measured in user beam with FC } \\
\text { chamber }\end{array}$ \\
\hline
\end{tabular}

Table 2 - relative standard uncertainties for parameters used in this COP, added in quadrature to give total uncertainty, with coverage factor $k=1$ unless otherwise stated.

\section{Appendix IV. Ion recombination corrections}

The correction for ion recombination is the sum of two components, initial recombination, which is independent of dose rate, and general (or volume) recombination, which is dose rate dependent and can be substantial for high-dose rate beams such as FFF. For continuous radiation, such as from a ${ }^{60} \mathrm{Co}$ source, initial recombination is dominant, but the correction is small and may usually be neglected. For pulsed radiation, general recombination is dominant and depends on the dose per pulse at the chamber, rather than the nominal machine dose rate.

NPL calibration coefficients relate to conditions of zero ion recombination, so all subsequent readings should be corrected, unless the difference is negligible $(\leq 0.1 \%)$. Correction factors 
can be determined experimentally using the two-voltage method. This method assumes a linear relationship between the reciprocal of the measured charge reading $(1 / \mathrm{M})$, and the reciprocal of the applied voltage $(1 / \mathrm{V})$, which may be verified using a full Jaffé plot (Scott and Greening 1963). For ion recombination correction factors, $k_{i o n}$, less than $3 \%$, the value may be approximated to within $0.1 \%$ using:

$$
k_{\text {ion }}=1+\frac{\left(M_{1} / M_{2}\right)^{-1}}{\left(V_{1} / V_{2}\right)^{-1}}
$$

where $M_{1}$ is the reading at the normal calibration voltage $V_{1}$, and $M_{2}$ is the reading at the reduced voltage $V_{2}$, measured using otherwise identical conditions. The ratio of the two voltages should be at least 3 . The upper voltage value should be selected to be below the charge multiplication region and at least as high as the voltage used for calibration measurements.

Alternatively, the following empirical relationships may be used for the following chamber and voltage combinations in static fields of pulsed radiation, with the two terms representing initial and general recombination respectively:

- NPL2611 chamber at $200 \mathrm{~V}: k_{\text {ion }}=1.0014+0.23 p$

- $\mathrm{A} 1 \mathrm{SL}$ chamber at $300 \mathrm{~V}: \quad k_{\text {ion }}=1.0001+0.04 p$

where $p$ is the dose per pulse (in cGy) at the chamber, which may be estimated from the nominal dose rate (MU. $\left.\mathrm{min}^{-1}\right)$, dose calibration (cGy.MU ${ }^{-1}$ ) and pulse repetition frequency (PRF, $\mathrm{Hz}$ ); from Burns et al. (1990) and Palmans et al. (2014) respectively. For example, a 6FFF beam at $1400 \mathrm{MU} \cdot \mathrm{min}^{-1}$, PRF $360 \mathrm{~Hz}$ (or pulse time $2.78 \mathrm{~ms}$ ) and $1 \mathrm{cGy} \cdot \mathrm{MU}^{-1}$ would have a dose per pulse of $0.065 \mathrm{cGy}$, and $\mathrm{k}_{\text {ion }}$ (NPL2611 chamber) of 1.016. These formulae have shown good agreement $(\leq 0.1 \%)$ in the experience of the working party members, including FFF beams, however, some verification with local measurement is recommended.

Ion recombination will vary with depth in phantom and SCD, especially for FFF beams where values are larger, therefore measurements of $\mathrm{TPR}_{20,10}$ should be corrected for local recombination conditions. Under these conditions, general recombination dominates, so the total correction may be assumed to be proportional to nominal dose rate at depth and correction factors can be scaled accordingly. However, for lower dose rates (<0.11 cGy per pulse) and TPR $_{20,10}$ 0.63-0.72 (i.e. both Varian and Elekta 6MV FFF and 10MV FFF beams), the experience of the working party is that ion recombination factors at the two different depths do not vary substantially (for both NPL2611 and Farmer-type chambers), and the impact on $\mathrm{N}_{\mathrm{D}, \mathrm{w}}$ is negligible $(<0.1 \%)$. 
For non-uniform beams (e.g. FFF), if the cross-calibration is performed using side-by-side simultaneous irradiation, then the difference in off-axis dose rate (and hence ion recombination) must be considered when applying chamber calibration coefficients to other positions, such as on central axis. In practice, if the dose-rate at the required measurement point is within $95 \%$ of the cross-calibration point then the relative change in ion recombination will be insignificant.

Charge multiplication is negligible for the NPL2611 at $200 \mathrm{~V}$ and Farmer-type chambers below $400 \mathrm{~V}$, but may be significant for small volume chambers operated at high voltages. However, since this effect is independent of dose rate, any effects will be incorporated into the crosscalibration, provided the FC is operated at the same voltage. Therefore, no specific guidance is given on recommended voltages for different chamber types, other than maintaining the same value for a specific chamber throughout the calibration process as much as possible.

\section{Appendix V. Recommendations for chamber positioning and calibration of linac output}

Appropriate positioning of the ionisation chamber for reference dosimetry involves both dose measurement and machine calibration considerations. Ideally these two setups would be the same, to avoid additional factors (with their own uncertainties) to convert between them.

At one level, the setup for machine calibration does not matter, so long as it is consistent between the treatment units and treatment planning system (TPS), staff are trained to follow the appropriate method, and appropriate independent checks are performed when adjusting machine calibration. However, consistency between centres has led to low levels of variation in reference dosimetry across the UK, and perhaps the most robust national reference dosimetry system in the world (Thomas et al. 2017).

For measurement of reference dose the recommended setup is a full scatter water-equivalent phantom. The phantom should extend at least $5 \mathrm{~cm}$ outside the field edges and $5 \mathrm{~cm}$ beyond the chamber along the beam axis. Smaller phantoms may be acceptable for constancy checks, but any differences to full scatter conditions should be established.

The recommended depth between the phantom surface and the chamber position depends on the quality index: for $0.57-0.75\left({ }^{60} \mathrm{Co}\right.$ to nominal $\left.10 \mathrm{MV}\right)$, the depth should be $5-10 \mathrm{~cm}$; and for $0.76-0.80$ (nominal $>10 \mathrm{MV}$ ) the depth should be $7-10 \mathrm{~cm}$. These ranges are based on the values given in the 1990 COP (5 cm and $7 \mathrm{~cm}$, Lillicrap et al. 1990), subsequent addenda (5 cm, Thomas et al. 2014 and Budgell et al. 2016) and in TRS398 (5 cm or $10 \mathrm{~cm}$, IAEA 2000) or TRS483 (10 cm, IAEA 2017). Chamber calibration coefficients may be used at measurement depths of $5-10 \mathrm{~cm}$ with negligible differences (Almond et al. 1999). Smaller depths may be 
affected by electron contamination and larger depths are likely to be impractical in terms of size of phantom and low signal at the chamber.

For machine calibration, an isocentric setup ${ }^{8}$ is preferred over a fixed SSD setup ${ }^{9}$ because of clinical relevance, consistency, and simplicity

- Calibration setup should be consistent with clinical treatments (IAEA 2000). Almost all current treatments are planned and delivered isocentrically, rather than the historical approach of changing the patient position to achieve a constant SSD. Fixed SSD treatments are in fact impossible with many modern units designed for rotational No additional depth dose factor is required to convert to a different calibration reference position (e.g. to $d_{\max }$ for fixed SSD approaches), which comes with added uncertainties and risk of error. It must be acknowledged that, despite the advantages of isocentric reference conditions, there is a level of risk in changing existing reference conditions within a clinic. The pros and cons are discussed in detail by Van den Heuvel et al (2018). It is the view of this working party that the advantages outweigh the disadvantages and hence only isocentric reference conditions are recommended in this COP.

In changing a centre's calibration conditions from fixed SSD to isocentric it is important that such a change is managed to ensure minimal clinical disruption. Appropriate times for making a change could include: new equipment which will require a separate beam model in the TPS; change of location, new satellite centre, or other break in service. It is not within the scope of this COP to provide detailed guidance on such a change, however the following list, although not exhaustive, may provide some initial points to consider:

\footnotetext{
${ }^{8}$ Isocentric calibration is where the chamber is positioned at a fixed distance, for example $100 \mathrm{~cm}$, from the source (coincident with the machine rotation isocentre), with a measurement depth in the phantom of for example $5 \mathrm{~cm}$ or $10 \mathrm{~cm}$. Calibration is then to a reference value (e.g. $1 \mathrm{cGy}$ per $\mathrm{MU}$ or another fixed value) at that same point.

${ }^{9}$ Fixed SSD calibration is where the source-to-surface distance (SSD) is set to a fixed value, for example $100 \mathrm{~cm}$, and the chamber is positioned a further $5 \mathrm{~cm}$ deep in the phantom. Usually, a percentage depth dose factor is then applied to calculate the dose at the depth of dose maximum $\left(\mathrm{d}_{\max }\right)$ and the unit calibrated to give a dose of

$1 \mathrm{cGy}$ per MU at $\mathrm{d}_{\max }$.
}

Deleted: (further discussion may be found in Van den Heuvel et al. (2018)): 
- Check recombination figures for new set-up (compare with dose per pulse at shorter SSD)

- Confirm calibration factors for smaller field size at measurement point

- Is it necessary to re-measure scatter factors?

- Is it necessary to generate new reference figures for quality control (QC) measurements?

- Recalibrate constancy devices accordingly

- Consider calibration processes for other devices used for relative dosimetry (e.g. portal imagers or arrays used for intensity modulated radiotherapy, IMRT QC)

- Possible creation of new beam models for independent MU calculation software or invivo dosimetry systems

- Dual-planning of patients for treatments before and after recalibration (MU recalculation)

- Training of staff on new QC procedures and manual MU calculations

- Consideration of holistic checks (e.g. patient-specific QC) at the end of process

Two options for machine calibration are recommended, since the working party felt both had merit depending on the local preference:

1. Isocentric setup, depth of $5 \mathrm{~cm}$ (up to $10 \mathrm{MV}$ ) or $7 \mathrm{~cm}(>10 \mathrm{MV}$ ) and calibration reference value for all energies of $1 \mathrm{cGy}$ per $\mathrm{MU}$;

2. Isocentric setup, depth of $10 \mathrm{~cm}$ (all energies) and calibration reference value of $1 \mathrm{cGy}$ per MU or a value per energy less than $1 \mathrm{cGy}$ per MU (e.g. $0.83 \mathrm{cGy}$ per MU at $10 \mathrm{~cm}$ deep, which is close to $1 \mathrm{cGy}$ per MU at $\mathrm{d}_{\max }$ for a Varian Truebeam $10 \mathrm{MV}$ beam).

Option 1 has the benefits of matching the setup at NPL for SS calibration, consistency with the typical cross-calibration setup, and consistency with the depths recommended in the previous 1990 COP and addenda for Tomotherapy and FFF beams. Retaining a calibration value of 1 cGy per MU allows for simple evaluation of any variations (e.g. $1.013 \mathrm{cGy}$ per $\mathrm{MU}$ is $1.3 \%$ higher than baseline) and equality of the number of $M U$ with dose in cGy in reference conditions. This approach keeps the same reference value of unity for all energies but requires depth change for higher energies to $7 \mathrm{~cm}$ (i.e. adding an extra block on top for solid water phantoms). Many centres only have lower energies (up to $10 \mathrm{MV}$ ), especially for units delivering mostly IMRT. In this case option 1 equates to a single depth of $5 \mathrm{~cm}$, which will be sufficient for many centres. $5 \mathrm{~cm}$ depth is allowed within TRS398 for centres wishing to maintain consistency with cobalt- 60 calibration, as used by most other standards laboratories, therefore a similar philosophy would be option 1 in this COP which matches the NPL setup. Use of reference 
values close to but not equal to $1 \mathrm{cGy}$ per $\mathrm{MU}$ are not recommended because of the risk of mis-application. For example, a $1 \%$ correction to dose-to-muscle has been variously and erroneously applied to centres in North America. Finally, it should be noted that the difference in machine output to deliver $1 \mathrm{cGy}$ per $\mathrm{MU}$ at $5 \mathrm{~cm}$ or $7 \mathrm{~cm}$ isocentric and at $d_{\max }$ fixed SSD is within $5-10 \%$, so large adjustments are not required.

Option 2 matches the setup recommended by TRS398 and TRS483, and also uses the same depth for both lower and higher energies. Use of a reference value lower than $1 \mathrm{cGy}$ per MU allows equality of the number of $\mathrm{MU}$ with dose in $\mathrm{CGy}$ in reference conditions as in historical fixed SSD methods ( $1 \mathrm{cGy}$ per MU at $\mathrm{d}_{\max }$ ). There may also be a benefit to maintaining similar typical MU values for safety checks, although new techniques such as IMRT will have already introduced step changes in expected values, and these have been implemented safety. Option 2 also keeps the same reference depth of $10 \mathrm{~cm}$ for all energies and treatment units and means that only a single extra measurement is required to produce an energy check. Some planning systems require additional data (e.g. output factors) defined at $10 \mathrm{~cm}$ deep. Finally, it should be noted that because this approach can maintain close to $1 \mathrm{cGy}$ per MU at $d_{\max }$, there is likely to be only a very minimal adjustment to the machine output to convert from a fixed SSD system to a $10 \mathrm{~cm}$ deep system. Some linac manufacturers do not recommend large changes from factory settings, which are avoided with this approach.

\section{Appendix VI. Recommendations for practical small field} measurements

There are significant differences between small and large fields that impact upon the ability to measure dose accurately as the field size is reduced. These differences are well described in publications such as IPEM Report 103 (IPEM 2010) and TRS483 (IAEA 2017) and can be summarised as:

- loss of CPE at the point of measurement;

- possible change in energy spectrum in the beam that may result in a changed detector response;

- the potentially significant perturbation of the radiation field by the detector.

810

Reference dosimetry for small fields is only necessary if the treatment unit is unable to achieve either the standard reference field size or an $m s r$ field that is sufficiently large that these small field problems do not arise. At the time of publication, TomoTherapy and CyberKnife cannot achieve the standard $10 \mathrm{~cm} \times 10 \mathrm{~cm}$ reference field but can achieve $m s r$ and pcsr fields large enough that it is not necessary to invoke small field reference dosimetry. The Gamma Knife, however, is a special case because it is unable to achieve a reference field size with dosimetric 
characteristics that are similar to a $10 \mathrm{~cm} \times 10 \mathrm{~cm}$ reference field. The Gamma Knife treatment fields are superpositions of multiple small fields and in this sense any definition of a reference field is a pcsr field. However, the simultaneous and mono-energetic nature of the delivery of the small beams making up the reference field means that it can be treated as a static $m s r$ rather than a composite or time-varying pcsr field.

Measurement of dose in small fields: In broad beam fields a calibrated detector may be used to measure dose in a range of fields that are different from the reference field. In small fields this cannot be assumed to be so. The detector used in reference dosimetry may perturb smaller beams to the extent that accurate dose measurements cannot be made simply with the reference dosimeter or cross-calibrated FC. Such field sizes are where the field extends less than the charged particle equilibrium range ( $r_{L C P E}$ ) beyond the outer boundary dimensions $(d)$ of the reference ion chamber i.e. full width half maximum (FWHM, or FFF beam equivalent) $\leq$

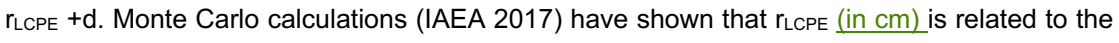
photon beam quality index by:

$$
r_{L C P E}=8.369 \times \mathrm{TPR}_{20,10}(10)-4.382
$$

In this situation, the dose should be calculated with the combination of the reference field dose and a field size factor and a chamber specific correction factor. The approach, for beam energies less than or equal to $10 \mathrm{MV}$, is well described in TRS483 and is summarised below. TRS483 combines the field size factor and the chamber specific correction factor into a single Field Output Factor (FOF) and the TRS483 nomenclature is retained here.

Field size calculation: In small fields $r_{L C P E}$ is not achieved and so the concept of equivalent field size based on equivalence of phantom scatter does not hold. TRS483 has taken the equivalent size of small fields $\left(s_{\text {clin }}\right)$ to be based on fields that exhibit the same detector perturbation factors which, for a given detector, can be equated to fields with the same area as the square field. Both TRS483 and IPEM103 take the borderline between the broad beam and the small field equivalent square field size methods for rectangular small fields where at least one dimension $A$ or $B$, is $\leq 4 \mathrm{~cm}$ and: $s_{\text {clin }}=\sqrt{A B}$, where $A$ and $B$ are the FWHM width of the beam. However, this is recommended only when the field is not very elongated and within the range $0.7<\frac{A}{B}<1.4$. Outside this range determination of FOF should be by measurement of the actual field size rather than by use of equivalent square field size. For circular small fields with diameter $\leq 4 \mathrm{~cm}$ and FWHM radius $\mathrm{r}$, the equivalent square field size $s_{\text {clin }}=r \sqrt{\pi}$.

Small field output factor calculation: The full scatter FOF $(\Omega)$ is given by the following equation: 
$\Omega_{Q_{c l i n}, Q_{m s r}}^{f_{c l i n}, f_{m s}}=\frac{M_{Q_{c l i n}}^{f_{c l i n}}}{M_{Q_{m s r}}^{f_{m s r}}} \boldsymbol{k}_{Q_{c l i n}, Q_{m s r}}^{f_{c l i n}, f_{m s r}}$

Note that when relating a small FOF to a standard $10 \mathrm{~cm} \times 10 \mathrm{~cm}$ reference field the msr in equation 23 can be replaced by $r e f$.

The detector specific output correction factor $\boldsymbol{k}_{\mathbf{Q}_{c l i n} \boldsymbol{Q}_{m s r}}^{f_{c l i n} f_{m s r}}$, used in small field dosimetry, accounts for the difference in response of the detector between the clinical and msr fields, and includes contributions from volume averaging effects, changes in response of the detector chamber to the changed spectra in the clinical field $\boldsymbol{f}_{\text {clin }}$ and charged particle fluence perturbation by the detector in the medium. That is:

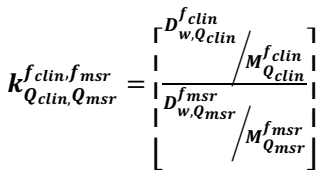

The full scatter FOF converts the absorbed dose to water in the reference field (ref or msr) to the absorbed dose to water in the clinical field $f_{\text {clin }}$. The detector specific output correction factor, $k_{Q_{c l i n} Q_{m s r}}^{f_{c l i n} f_{m s r}}$ is very dependent on the characteristics of the detector used and, where broad beam dosimetry conditions apply and where Farmer-like chambers are used, it is close to unity. However, where very small ion chambers or solid-state detectors are used for measurement in small field conditions it cannot be assumed to be unity. Values of $k_{Q_{c l i n} Q_{m s r}}^{f_{c l i n} f_{m s}}$ for a range of detectors are published in TRS483 as a function of therapy machine and field size and the user is referred to those and any subsequent updates for determination of appropriate values. Users should note the depth and field size for which the factors are valid and also note the published commentary on these data in Das and Francescon (2018). In-phantom FOFs should ideally be measured at the same depth as used for the reference dosimetry.

Small field output factor calculation using daisy-chaining: In situations where an ionisation chamber can be used to measure FOFs in larger fields but a smaller detector is required for the smallest fields, the FOFs can be linked using an intermediate field, a process sometimes termed daisy-chaining. In this method, an ionisation chamber, $I C$, is used in the smallest field that does not exhibit small field conditions, referred to as $f_{\text {int }}$, and at least one small field detector, det, is used both at $f_{\text {int }}$ and at smaller field sizes. The FOF $(\Omega)$ is described in TRS483 as:

$\Omega_{Q_{c l i n}, Q_{m s r}}^{f_{c l i n}, f_{m s}}=\left[\frac{M_{Q_{c l i n}}^{f_{\text {clin }}}}{M_{Q_{\text {int }}}^{f_{\text {int }}}} k_{Q_{c l i n}, Q_{\text {int }}}^{f_{\text {clin }}, f_{\text {int }}}\right] \operatorname{det}\left[\frac{M_{Q_{\text {int }}}^{f_{\text {int }}}}{M_{Q_{m s r}}^{f_{m s r}}} k_{Q_{\text {int }}, Q_{m s r}}^{f_{\text {int }}, f_{m s r}}\right]_{I C}$

This limits the influence of energy dependence of the small field detector on the FOF to only 
those field sizes where the small field detector should be used. It assumes that the shape of the FOF curve for the range of field sizes measured by the small field detector is correct and rescaling is required to emulate the response of the ionisation chamber. Tables 23-27 in TRS483 provide detector specific output correction factors, $k_{Q_{c l i n} Q_{r e f}}^{f_{c l i n} f_{r e f}}$, related to the reference

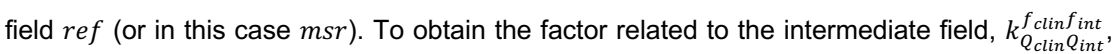
the ratio of clinical to intermediate field factors should be used:

$\left[k_{Q_{c l i n}, Q_{\text {int }}}^{f_{\text {clin }}, f_{\text {int }}}\right]_{\text {det }}=\frac{\left[\begin{array}{c}{ }^{f_{\text {clin }}, f_{\text {ref }}} \\ Q_{\text {clin }}, Q_{r e f}\end{array}\right]_{\text {det }}}{\left[{ }^{f_{\text {int }}, f_{\text {ref }}, Q_{\text {ref }}}\right]_{\text {det }}}$

The intermediate field, $f_{\text {int }}$, should not exhibit small field characteristics and so the correction factor for the ionisation chamber, $\left[k_{Q_{\text {int }} Q_{\text {ref }}}^{f_{\text {int }} f_{r C}}\right]_{I C}$, should be unity. Where $f_{\text {ref }}\left(\right.$ or $\left.f_{m s r}\right)$ is larger than $4 \mathrm{~cm}$ the NPL2611 and A1SL chambers can be used for reference dosimetry but when selecting a chamber for daisy-chaining FOFs any of the chambers listed in Tables 4 \& 5 of TRS483, and any subsequent addenda, can be selected.

\section{Measurement of small field output factors}

Measurements should be corrected for influence quantities, even for relative measurements, because temporal, thermal or dose rate corrections may change over the measurement sequence. All measurements in small fields should be corroborated by multiple measurements with at least 2 different detectors whose characteristics, such as energy dependency, dose rate dependency, volume averaging effects and polarity dependency on field size are understood as per the guidance in IPEM103 and TRS483.

Any hysteresis or inaccuracy in the collimation of small field sizes should also be accounted for if the field size can be reached from either a smaller or a larger opening position as small changes in the field size can result in relatively large changes in field area, and therefore in the FOF. Records of the measurement should make clear whether the field size used is that set by the collimating device (geometrical field size) or that measured at the depth of measurement (radiation field size). Detector specific output calibration factors, in TRS483, are given for the FWHM which should be measured with a detector that is not energy dependent or susceptible to volume averaging effects.

\section{Appendix VII. Worked examples for application of the COP}

To facilitate implementation of this code, four worked examples are presented to cover a 
- Treatment unit capable of $10 \mathrm{~cm} \times 10 \mathrm{~cm}$ reference field, flattened reference field (WFF), Table 3

- Treatment unit capable of $10 \mathrm{~cm} \times 10 \mathrm{~cm}$ reference field, unflattened reference field (FFF), Table 4

- Treatment unit not capable of $10 \mathrm{~cm} \times 10 \mathrm{~cm}$ reference field, TomoTherapy, Table $5^{10}$

Each example includes the following steps:

a) Calibration coefficient for the SS in the local beam;

b) Cross-calibration of SS and FC in the local beam;

c) Reference output measurement in the local beam.

925 All depths are quoted in water unless otherwise stated. A fourth example (Table 6) then describes the calculation of a small field output factor for an unflattened field on a treatment unit capable of $10 \mathrm{~cm} \times 10 \mathrm{~cm}$ reference field.

${ }^{10}$ N.B. For Cyberknife units, a similar approach may be taken to that shown in Table 5, with the following typical parameters: $6 \mathrm{MV}$ FFF, $80 \mathrm{~cm} \mathrm{SCD}, 800 \mathrm{MU} \cdot \mathrm{min}^{-1}$; field size $6 \mathrm{~cm}$ circle (equivalent square 5.4 $\mathrm{cm}$, or $5.0 \mathrm{~cm}$ as given in TRS483), TPR $20 / 10(10 \times 10) \sim 0.66$; unit is typically calibrated to give $1 \mathrm{cGy} . \mathrm{MU}^{-}$ ${ }^{1}$ at $d_{\max }$ depth (isocentric), so as not to change output too far from the factory value; $\operatorname{PDD}(5 \mathrm{~cm}) \sim 90 \%$, so expect value at $5 \mathrm{~cm}$ deep $\sim 0.90 \mathrm{cGy} \cdot \mathrm{MU}^{-1}$.

Deleted: Table 3Table 3

Formatted: Font: Not Bold

Deleted: Table 4Table 4

Formatted: Font: Not Bold

Formatted: Font: Not Bold

Deleted: Table 5Table 5

Deleted: Table 6 Table 6

Formatted: Font: Not Bold 


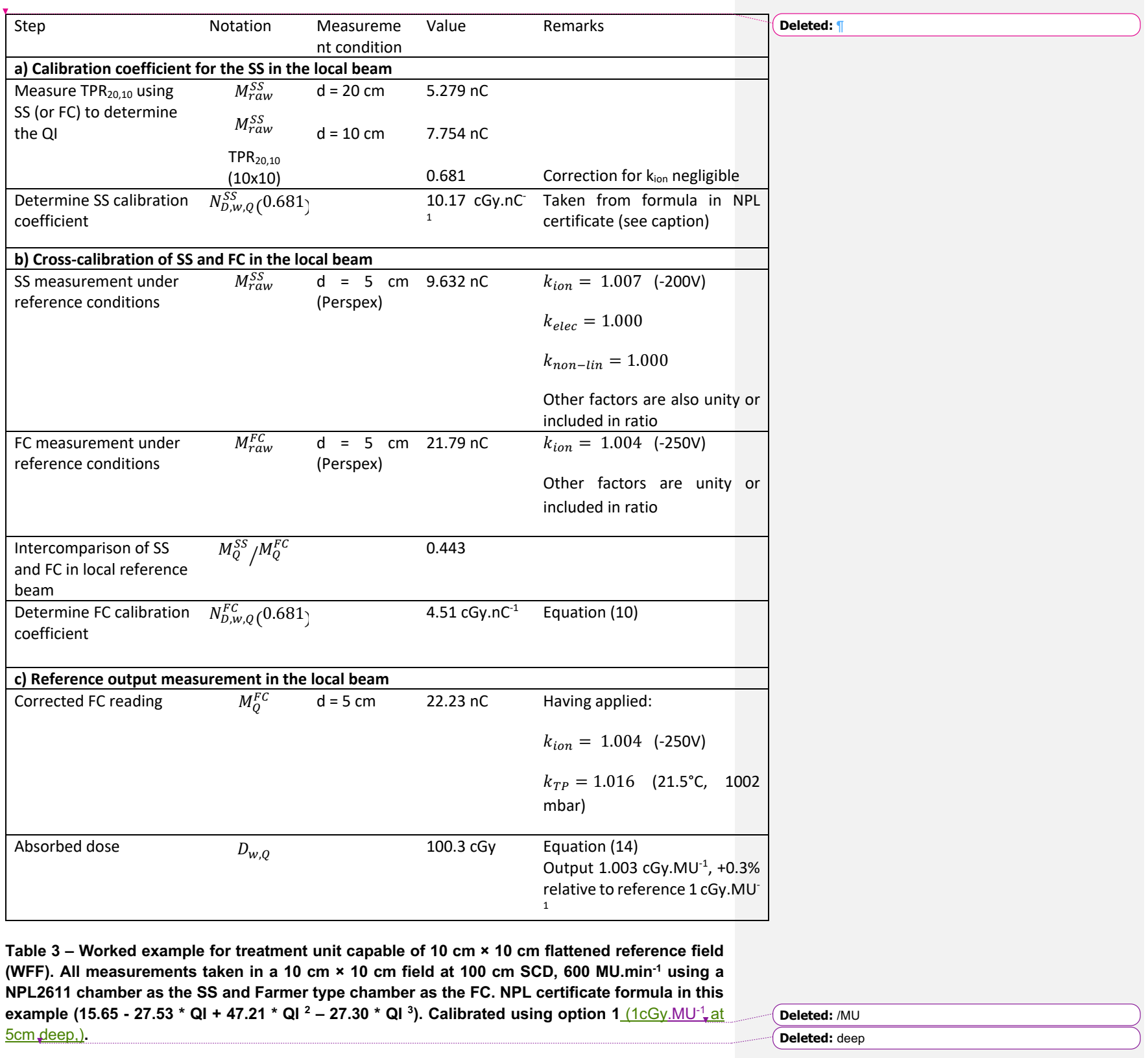




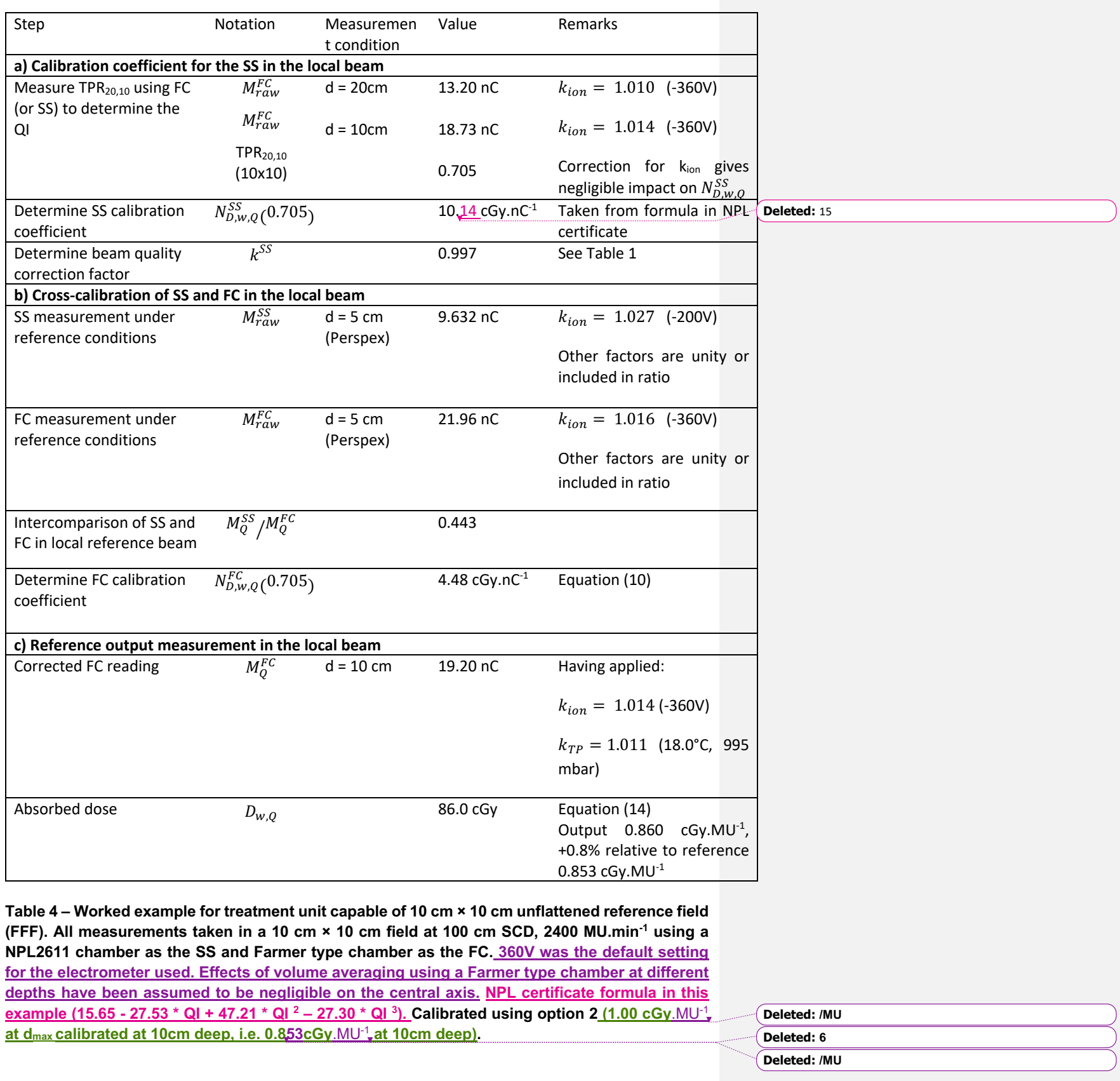




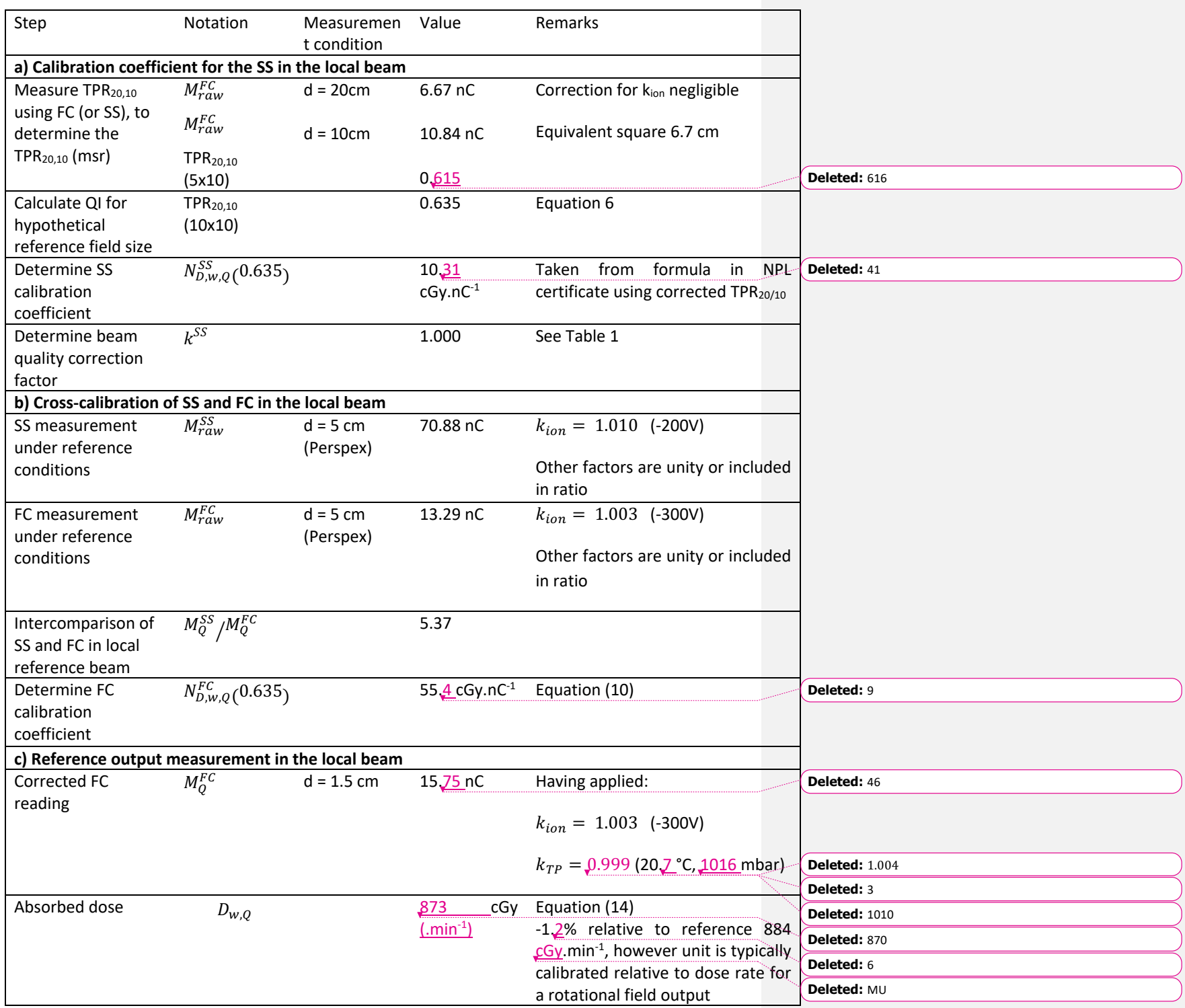

Table 5 - Worked example for TomoTherapy unit. All measurements taken in a static beam of field size $5 \mathrm{~cm} \times 10 \mathrm{~cm}$ at $85 \mathrm{~cm}$ SCD, $60 \mathrm{sec}$ exposure (1031 MU including $10 \mathrm{sec}$ ramp up), using 
a NPL2611 chamber as the SS and A1SL chamber as the FC. NPL certificate formula in this example $\left(15.79-27.78\right.$ * $\left.\mathrm{QI}+47.64{ }^{*} \mathrm{QI}^{2}-27.55{ }^{*} \mathrm{QI}{ }^{3}\right)$.

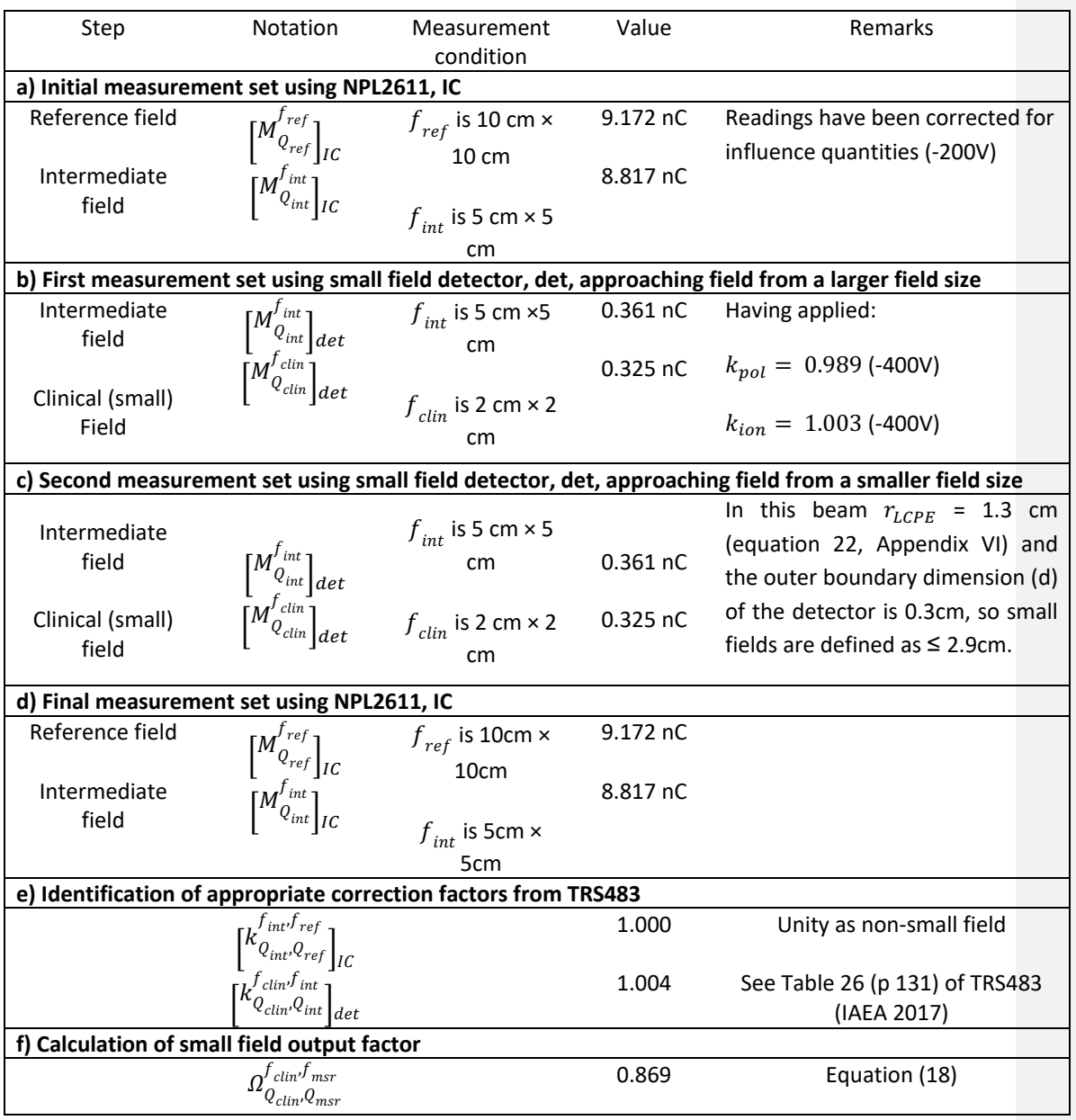

Table 6 - Calculation of a small field output factor using daisy-chaining for an unflattened field on a treatment unit capable of $10 \mathrm{~cm} \times 10 \mathrm{~cm}$ reference field. Reference field size is $10 \mathrm{~cm} \times 10$ $\mathrm{cm}$ at $100 \mathrm{~cm}$ SCD and $5 \mathrm{~cm}$ depth. IC used is NPL2611 and small field detector is PTW Pinpoint 3D 31016. 


\section{References}

Abdel-Rahman W, Evans M D C, Serré L, McCaffrey JP, Podgorsak EB and Seuntjens JP 2009 Clinic based transfer of the $\mathrm{N}_{\mathrm{D}, \mathrm{w}}{ }^{60} \mathrm{Co}$ calibration coefficient using a linear accelerator Med. Phys. 36 929-38

Alfonso R, Andreo P, Capote R, Huq MS, Kilby W, Kjäll P, Mackie TR, Palmans H, Rosser K, Seuntjens J, Ullrich W and Vatnitsky S 2008 A new formalism for reference dosimetry of small and nonstandard fields Med. Phys. 35 5179-86

Almond P, Biggs P J, Coursey B M, Hanson W F, Huq M S, Nath R and Rogers D W O 1999

AAPM's TG-51 protocol for clinical reference dosimetry of high-energy photon and electron beams Med. Phys. 26 1847-70

Andreo P 2015 Dose to 'water-like' media or dose to tissue in MV photons radiotherapy treatment planning: still a matter of debate. Phys. Med. Biol. 60 309-37

Andreo P and Brahme A 1986 Stopping power data for high energy photon beams. Phys. Med.

Biol. 31 839-58

Bass G A, Barry M A, Duane S, Homer M J, Kelly M, Manning J W, Maughan D J, Nutbrown R F, Sander T, Shipley D R and Snaith J A D 2019 Changes to the UK national primary standards of air kerma and absorbed dose incorporating ICRU Report 90 recommendations: NPL Report IR 55 (Teddington: National Physical Laboratory)

British Institute of Radiology (BIR) 1996 Central axis depth dose data for use in radiotherapy: BJR supplement 25 (London: BIR)

Budgell G, Brown K, Cashmore J, Duane S, Frame J, Hardy M, Paynter D and Thomas R 2016 IPEM topical report 1: guidance on implementing flattening filter free (FFF) radiotherapy Phys. Med. Biol. 61 8360-94

Burns J E 1994 Absorbed-dose calibrations in high-energy photon beams at the National Physical Laboratory: conversion procedure Phys. Med. Biol. 39 1555-75

Burns J E and Rosser K E 1990 Saturation Correction for the NE 2560/1 Dosemeter in Photon Dosimetry Phys. Med. Biol. 35 687-702

Castellano E A, Eaton D J, MacDougall N D and Cherry SR 2016 IPEM codes or practice and topical report series Phys. Med. Biol. 61 E5-6

Das I and Francescon P 2018 Comments on the TRS-483 protocol for small field dosimetry 
Med. Phys. 45 5666-8

Deutsches Institut fur Normung (DIN) 2008 DIN 6800-2 Procedures of dosimetry with probe type detectors for photon and electron radiation - Part 2: lonization chamber dosimetry of high energy photon and electron radiation https://www.beuth.de/en/standard/din-68002/105666743

Eaton D J, Bolton S, Thomas R A and Clark C H 2015a Inter-departmental dosimetry auditsdevelopment of methods and lessons learned J. Med. Phys. 40(4) 183

Eaton D J, Thomas R A S and Duane S 2015b Multi-centre audit of absolute dose calibration for flattening filter-free beams Biomed. Phys. Eng. Express 1(4) 047002

Jnternational Commission on Radiation Units and Measurements (ICRU) 2016 Key data for ionizing-radiation dosimetry: measurement standards and applications: ICRU Report $90 \mathrm{~J}$ ICRU 141 (Oxford: Oxford University Press)

Institute of Physics and Engineering in Medicine (IPEM) 2010 Small field MV photon dosimetry, Report 103 (York: IPEM)

Institute of Physics and Engineering in Medicine (IPEM) 2018 Physics Aspects of Quality Control for Radiotherapy, Report 81, $2^{\text {nd }}$ edition (York: IPEM)

International Atomic Energy Agency (IAEA) 2000 IAEA TRS398 Absorbed dose determination in external beam radiotherapy: an international code of practice for dosimetry based on standards of absorbed dose to water (Vienna: IAEA) http://wwwpub.iaea.org/mtcd/publications/pdf/trs398 scr.pdf

International Atomic Energy Agency (IAEA) 2017 IAEA TRS483. Dosimetry of Small Static Fields Used in External Beam Radiotherapy: An International Code of Practice for Reference and Relative Dose Determination (Vienna: IAEA) https://wwwpub.iaea.org/MTCD/Publications/PDF/D483 web.pdf

Kinoshita N, Kohno R and Oguchi H 2019 Influence of entrance window deformation on reference dosimetry measurement in various beam modalities Med. Phys. 46 1037-43

Lillicrap S C, Owen B, Williams J R and Williams P C 1990 Code of practice for high-energy photon therapy dosimetry based on the NPL absorbed dose calibration service Phys. Med. Biol. 35 1355-60

Ma C-M and Li J 2011 Dose specification for radiation therapy: dose to water or dose to medium? Phys. Med. Biol. 56 3073-89
Deleted: $f$

Deleted: Huq M S, Hwang M-S, Teo T P, Young Jang S, Heron D E and Lalonde R J 2018 A dosimetric evaluation of the IAEA-AAPM TRS483 code of practice for dosimetry of small static fields used in conventional linac beams and Addendum protocols Med. Phys. 45 4257-73T 
McEwen M, DeWerd L, Ibbott G, Followill D, Rogers D W O, Seltzer S and Seuntjens J 2014 Addendum to the AAPM's TG-51 protocol for clinical reference dosimetry of high-energy photon beams Med. Phys. 41041501

Morgan A M, Aird E G A, Aukett R J, Duane S, Jenkins N H, Mayles W P M, Moretti C and Thwaites D I 2000 IPEM guidelines on dosimeter systems for use as transfer instruments between the UK primary dosimetry standards laboratory (NPL) and radiotherapy centres Phys.

Med. Biol. 45 2445-57

Nederlandse Commissie voor Stralingsdosimetrie (NCS) 2012 NCS 18: Code of Practice for the Absorbed Dose Determination in High Energy Photon and Electron Beams https://radiationdosimetry.org/ncs/documents/ncs-18-cop-dosimetry-in-water

Nutbrown R F, Duane S, Shipley D R and Thomas R A S 2002 Evaluation of factors to convert absorbed dose calibrations from graphite to water for the NPL high-energy photon calibration service Phys. Med. Biol. 47 441-54

Palmans H 2012 Determination of the beam quality index of high-energy photon beams under nonstandard reference conditions Med. Phys. 39 5513-9

Palmans H, Thomas R A S, Duane S, Sterpin E and Vynckier S 2010 Ion Recombination for Ionization Chamber Dosimetry in a Helical Tomotherapy Unit Med. Phys. 37 2876-89

Pearce J A D, Shipley D R and Duane S 2011 Transfer of the UK absorbed dose primary standard for photon beams from the research linac to the clinical linac at NPL Metrologia 48 $365-74$

Scott P B and Greening J R 1963 The Determination of Saturation Currents in Free-Air Ionization Chambers by Extrapolation Methods Phys. Med. Biol. 8 51-8

Thomas R A, Bolt M A, Bass G, Nutbrown R, Chen T, Nisbet A and Clark C H 2017 Radiotherapy reference dose audit in the United Kingdom by the National Physical Laboratory: 20 years of consistency and improvements Phys. Imag. Radiat. Oncol. 3 21-27

Thomas S J, Aspradakis M M, Byrne J P, Chalmers G, Duane S, Rogers J, Thomas R A S, Tudor G S J and Twyman N 2014 Reference dosimetry on TomoTherapy: an addendum to the 1990 UK MV dosimetry code of practice Phys. Med. Biol. 59 1339-52

Van den Heuvel F, Wu Q and Cai J 2018 In modern linacs monitor units should be defined in water at $10 \mathrm{~cm}$ depth rather than at $\mathrm{d}_{\max }$ Med. Phys. 45 4789-92 